\title{
OPEN Multiple myeloma hinders erythropoiesis and causes anaemia owing to high levels of CCL3 in the bone marrow microenvironment
}

\author{
Lanting Liu ${ }^{1,6}$, Zhen $\mathrm{Yu}^{1,6}$, Hui Cheng ${ }^{1}$, Xuehan $\mathrm{Mao}^{1}$, Weiwei Sui ${ }^{1}$, Shuhui Deng ${ }^{1}$, \\ Xiaojing Wei ${ }^{1}$, Junqiang $\mathrm{Lv}^{2}$, Chenxing $\mathrm{Du}^{1}$, Jie Xu ${ }^{1}$, Wenyang Huang ${ }^{1}$, Shuang $\mathrm{Xia}^{3}$, Gang $\mathrm{An}^{1}$, \\ Wen Zhou ${ }^{4}$, Xiaoke Ma ${ }^{5 凶}$, Tao Cheng ${ }^{1}$, Lugui Qiu ${ }^{1}$ \& Mu Hao ${ }^{1 凶}$
}

Anaemia is the most common complication of myeloma and is associated with worse clinical outcomes. Although marrow replacement with myeloma cells is widely considered a mechanistic rationale for anaemia, the exact process has not been fully understood. Our large cohort of 1363 myeloma patients had more than $50 \%$ of patients with moderate or severe anaemia at the time of diagnosis. Anaemia positively correlated with myeloma cell infiltration in the bone marrow (BM) and worse patient outcomes. The quantity and erythroid differentiation of HSPCs were affected by myeloma cell infiltration in the BM. The master regulators of erythropoiesis, GATA1 and KLF1, were obviously downregulated in myeloma HSPCs. However, the gene encoding the chemokine CCL3 showed significantly upregulated expression. Elevated CCL3 in the BM plasma of myeloma further inhibited the erythropoiesis of HSPCs via activation of CCL3/CCR1/p38 signalling and suppressed GATA1 expression. Treatment with a CCR1 antagonist effectively recovered GATA1 expression and rescued erythropoiesis in HSPCs. Myeloma cell infiltration causes elevated expression of CCL3 in BM, which suppresses the erythropoiesis of HSPCs and results in anaemia by downregulating the level of GATA1 in HSPCs. Thus, our study indicates that targeting CCL3 would be a potential strategy against anaemia and improve the survival of myeloma patients.

Multiple myeloma (MM) is characterized by bone destruction, anaemia, and renal and immunological impairment. These complications may lead to a severe reduction in the quality of life of myeloma patients and may shorten their life expectancy ${ }^{1,2}$. Anaemia is the most common complication of myeloma patients at diagnosis and in almost all patients with uncontrolled disease. Given the known adverse impact of multiple myeloma on physical functioning and quality-of-life variables, including fatigue and cognitive function, managing anaemia should be an integral part of myeloma patient care. Elucidating the mechanism underlying anaemia and developing an effective treatment are critical for improving the quality of life of MM patients.

The most frequent underlying pathophysiological conditions reported previously in myeloma-related anaemia are aberrant iron metabolism, renal impairment and anaemia of chronic disease $\mathrm{s}^{3-5}$. In patients who achieve complete remission after chemotherapy, their anaemia usually normalizes. Non-responders and relapsed myeloma

\footnotetext{
${ }^{1}$ State Key Laboratory of Experimental Hematology, National Clinical Research Center for Blood Diseases, Institute of Hematology and Blood Diseases Hospital, Chinese Academy of Medical Sciences and Peking Union Medical College, Tianjin 300020, China. ${ }^{2}$ Department of Immunology, Key Laboratory of Immune Microenvironment and Disease of the Educational Ministry of China, Tianjin Key Laboratory of Cellular and Molecular Immunology, School of Basic Medical Sciences, Tianjin Medical University, Tianjin 300070, China. ${ }^{3}$ Department of Radiology, Tianjin First Central Hospital, Tianjin 300192, China. ${ }^{4}$ Cancer Research Institute, Key Laboratory of Carcinogenesis and Cancer Invasion, Ministry of Education; Key Laboratory of Carcinogenesis, National Health and Family Planning Commission, Central South University, Hunan, China. ${ }^{5}$ School of Computer Science and Technology, Xidian University, Xi'an, China. ${ }^{6}$ These authors contributed equally: Lanting Liu and Zhen Yu. ${ }^{\boxplus}$ email: xkma@ xidian.edu.cn; haomu@ihcams.ac.cn
} 


\begin{tabular}{|l|l|l|l|l|}
\hline Characteristics & All patients (\%) & Hgb $>120$ g/L (\%) & Hgb $\leq 120$ g/L (\%) & $p$ value \\
\hline No. of patients & 1363 & $213(15.6 \%)$ & $1150(84.4 \%)$ & \\
\hline Gender & $839(61.6 \%)$ & $166(77.9 \%)$ & $673(58.5 \%)$ & \\
\hline Male & $524(38.4 \%)$ & $47(22.1 \%)$ & $477(41.5 \%)$ & \\
\hline Female & \multicolumn{5}{|l|}{} & \\
\hline Age & $1016(74.5 \%)$ & $178(83.6 \%)$ & $838(72.9 \%)$ & \\
\hline$<65$ year & $347(25.5 \%)$ & $35(16.4 \%)$ & $312(27.1 \%)$ & \\
\hline$\geq 65$ year & \multicolumn{5}{|l|}{} & \\
\hline Ig isotype & $654(48.5 \%)$ & $94(47.0 \%)$ & $560(48.7 \%)$ & \\
\hline IgG & $317(23.5 \%)$ & $52(26.0 \%)$ & $265(23.1 \%)$ & \\
\hline IgA & $65(4.8 \%)$ & $8(4.0 \%)$ & $57(5.0 \%)$ & \\
\hline IgD & $272(20.2 \%)$ & $38(19.0 \%)$ & $234(20.4 \%)$ & \\
\hline Light chain & $41(3.0 \%)$ & $8(4.0 \%)$ & $33(2.8 \%)$ & \\
\hline Non-secretory & & & & $<0.0001$ \\
\hline RISS stage & $84(13.5 \%)$ & $49(40.2 \%)$ & $35(6.3 \%)$ & \\
\hline I & $389(55.8 \%)$ & $56(45.9 \%)$ & $333(59.7 \%)$ & \\
\hline II & $207(30.7 \%)$ & $17(13.9 \%)$ & $190(34.0 \%)$ & \\
\hline III & & & & $<0.0001$ \\
\hline DS stage & $86(6.5 \%)$ & $50(25.1 \%)$ & $36(3.2 \%)$ & \\
\hline I & $122(9.2 \%)$ & $29(14.6 \%)$ & $93(8.3 \%)$ & \\
\hline II & $1117(84.3 \%)$ & $120(60.3 \%)$ & $997(88.5 \%)$ & \\
\hline III & $29.5(0-98.5)$ & $9(0-91.5)$ & $33(0-98.5)$ & $<0.0001$ \\
\hline BM-infiltration (\%) median; range & & & \\
\hline MBD & $47(31.5 \%)$ & $12(60.0 \%)$ & $35(27.1 \%)$ & \\
\hline $0-2$ & $102(68.5 \%)$ & $8(40.0 \%)$ & $94(72.9 \%)$ & \\
\hline $3-4$ & $89(4-197)$ & $132(121-197)$ & $81(4-120)$ & \\
\hline Hemoglobin (g/L) median; range & & & \\
\hline & & & \\
\hline
\end{tabular}

Table 1. Baseline characteristics of 1363 NDMM patients. RISS Revised international staging system, DS Durie Salmon, $M B D$ myeloma bone disease.

patients often continue to suffer from anaemia ${ }^{6}$. This means that myeloma cell infiltration is highly involved in the pathogenesis of anaemia in myeloma patients. However, the mechanism of anaemia in myeloma is not fully understood.

Bone marrow is the main location where the growth and differentiation of myeloma cells and haematopoietic stem and progenitor cells (HSPCs) ${ }^{7,8}$. Specialized bone marrow niches control the homeostasis and differentiation of HSPCs. An aberrant microenvironment comprising myeloma cell infiltration and increased concentrations of soluble cytokines, growth factors derived from malignant cells and innocent bystander cells is beneficial for myeloma cell survival and plays a pivotal role in controlling HSPC differentiation ${ }^{9-12}$. Based on this, we focused on the effects and molecular mechanisms of myeloma cell infiltration in the bone marrow resulting in defective erythropoiesis of HSPCs and anaemia in the present study.

Here, we demonstrated that the quantity and erythroid differentiation of HSPCs were affected by myeloma cell infiltration in the bone marrow microenvironment. Most HSPCs were arrested in G0 phase of the cell cycle and exhibited reduced proliferation towards megakaryocyte-erythroid progenitors. We also found that the expression of the transcription factors GATA1 and KLF1 was significantly downregulated in the HSPCs of myeloma patients, especially after the cells were co-cultured with bone marrow plasma from myeloma patients. Further analysis demonstrated that the increase in the levels of the chemokine CCL3 in bone marrow plasma effectively blocks the erythropoiesis of HSPCs via downregulation of GATA1 in HSPCs by activating the p38 signalling pathway. Inhibition of CCL3 activity with a CCR1 antagonist efficiently restored the expression of GATA1 and rescued the erythropoiesis of HSPCs. These findings suggest that the aberrant microenvironment owing to myeloma cells suppressed the differentiation of HSPCs to erythrocytes. The chemokine CCL3 caused not only lytic bone lesions but also defective erythropoiesis. Targeting CCL3 is a potential strategy against anaemia and bone disease, which means killing two birds with one stone in patients with multiple myeloma.

\section{Results}

Anaemia is a striking symptom that predicts worse outcome in myeloma patients. Newly diagnosed MM (NDMM) patients $(n=1363)$ were enrolled in this analysis. The patients' clinical features are described in Table 1 . Our data demonstrated that $84.4 \%$ of patients $(1150 / 1363)$ had anaemia $(\mathrm{Hgb}<120 \mathrm{~g} / \mathrm{L})$ at the time of diagnosis regardless of sex, and 55.2\% (753/1363) of patients had moderate (90-120 g/L) or severe anaemia (60-90 g/L) (Fig. 1A,B) at the time of diagnosis. We also found that the reduction in Hgb was positively correlated with myeloma cell burden. The patients with advanced stage disease had lower levels of Hgb both in the DS (Durie-Salmon) staging system $(80.1 \pm 0.8 \mathrm{~g} / \mathrm{L}$ in DS stage III) and the Revised International Staging 
A.

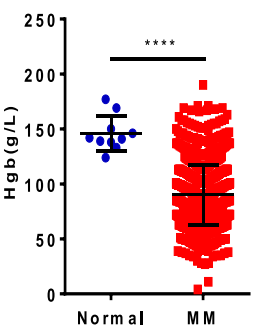

B.

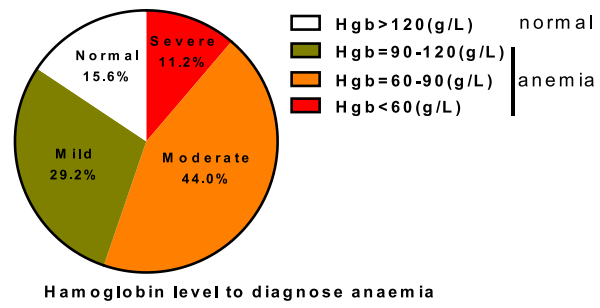

C.

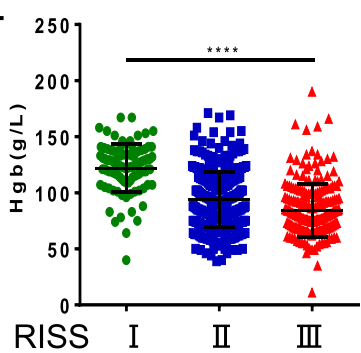

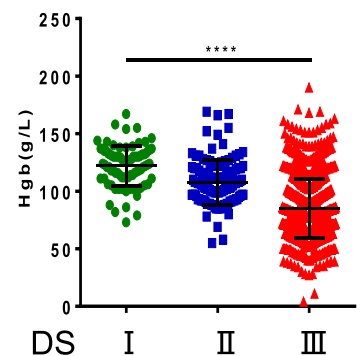

D.

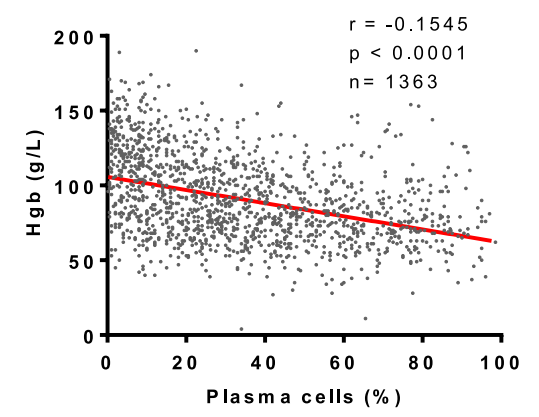

E.

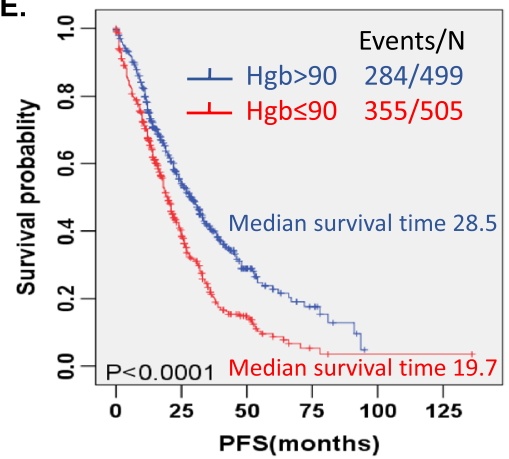

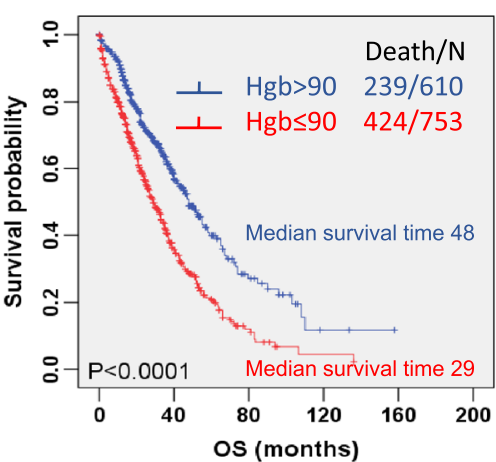

Figure 1. Anaemia is an important symptom of myeloma and correlates with worse outcomes in myeloma patients. (A) The clinical data of a large cohort of myeloma patients showed the levels of haemoglobin in normal donors $(\mathrm{n}=10)$ and NDMM patients $(\mathrm{n}=1363)$. (B) The clinical analysis showed that $84.4 \%(1150 / 1363)$ of NDMM patients had anaemia at diagnosis, and $55.2 \%(753 / 1363)$ of patients had moderate $(90-120 \mathrm{~g} / \mathrm{L})$ or severe (60-90 g/L) anaemia. (C) The decrease in haemoglobin in NDMM patients was positively correlated with the disease stage. R-ISS stage (left) or DS stage (right). (D) The levels of haemoglobin in NDMM patients were negatively correlated with myeloma cell infiltration in the bone marrow $(\mathrm{r}=-0.1545, p<0.0001, \mathrm{n}=1363)$. (E) Kaplan-Meier analysis showed shorter survival of myeloma patients with decreased haemoglobin levels.

System (RISS) $(84.3 \pm 1.6 \mathrm{~g} / \mathrm{L}$ in R-ISS stage III; Table 1 and Fig. 1C). In particular, the level of Hgb in myeloma patients was negatively correlated with the infiltration of myeloma cells in the bone marrow $(\mathrm{r}=-0.1545$, $p<0.0001$; Fig. 1D). Moreover, patients with anaemia had more severe bone disease, as indicated in Table 1. Kaplan-Meier analysis indicated that the survival of patients with lower levels of $\operatorname{Hgb}(\mathrm{n}=753, \mathrm{Hgb} \leq 90 \mathrm{~g} / \mathrm{L})$ was shorter than that of patients with higher levels of $\mathrm{Hgb}(\mathrm{n}=610, \mathrm{Hgb}>90 \mathrm{~g} / \mathrm{L})$. Progression-free survival (PFS) was 19.7 vs. 28.5 months $(p<0.0001)$, and the overall survival (OS) was 29.0 vs. 48.0 months $(p<0.0001)$ between the low Hgb group and high Hgb group, respectively (Fig. 1E). If the cut-off Hgb level was $60 \mathrm{~g} / \mathrm{L}$, the survival of patients with severely decreasing Hgb levels $(\leq 60 \mathrm{~g} / \mathrm{L})$ was further shortened compared to that of patients with Hgb levels greater than $60 \mathrm{~g} / \mathrm{L}$ (Supplementary Figure 2). Our findings clearly demonstrated that anaemia is a striking symptom and efficiently predicted worse outcomes of patients with myeloma. Specifically, Hgb levels were negatively correlated with the infiltration of myeloma cells.

Myeloma bone marrow suppresses the growth and differentiation of HSPCs. Flow cytometry analysis revealed that the population of $\mathrm{CD} 45^{+} \mathrm{CD} 34^{+} \mathrm{HSPCs}$ was significantly decreased in bone marrow biopsy samples of myeloma patients compared to the healthy controls $(0.43 \% \pm 0.04 \%$ vs. $1.84 \% \pm 0.17 \%, p<0.0001$; Fig. 2A,B). The number of $\mathrm{CD} 45^{+} \mathrm{CD} 34^{+} \mathrm{HSPCs}$ was negatively correlated with the number of $\mathrm{CD} 38^{+} \mathrm{CD} 138^{+}$ myeloma cells in the bone marrow of MM patients $(r=-0.6112, p<0.0001$; Fig. 2C). As Fig. 2D shows, the

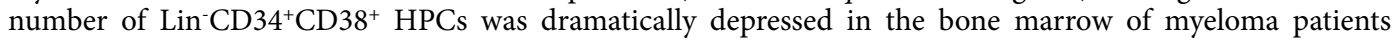
compared with that of healthy patients $(0.39 \% \pm 0.07 \%$ vs. $0.98 \% \pm 0.14 \%, p=0.0002)$. However, the number of $\mathrm{Lin}^{-} \mathrm{CD} 34^{+} \mathrm{CD} 38^{-}$HSCs did not show an obvious decrease between the two groups $(0.09 \% \pm 0.02 \%$ vs. $0.12 \% \pm 0.02 \%)$. The data indicated that the decrease in HSPCs $\left(\mathrm{Lin}^{-} \mathrm{CD} 34^{+}\right.$) was mainly due to the differentiation defects of HSCs, which caused a decrease in the HPC (haematopoietic progenitor cells, $\operatorname{Lin}^{-} \mathrm{CD} 34^{+} \mathrm{CD} 38^{+}$) population but not the HSC (haematopoietic stem cells, $\mathrm{Lin}^{-} \mathrm{CD} 34^{+} \mathrm{CD} 38^{-}$) population.

To confirm these results, the C57BL/KaLwRij murine myeloma model was utilized. Six weeks after injection of the 5 TGM1 mouse myeloma cell line $\left(2 \times 10^{6} / \mathrm{mouse}\right)$, the elevated CD138 ${ }^{+}$mouse myeloma cell population $(10.9 \% \pm 1.2 \%, \mathrm{n}=6)$ and IgG2b levels $(451 \pm 10 \mu \mathrm{g} / \mathrm{mL}, \mathrm{n}=6)$ in the bone marrow of MM-5TGM1 mice indicated the successful proliferation of myeloma cells (Supplementary Figure 3A\&3B). Routine tests of peripheral blood revealed a significant decrease in Hgb levels in all mice injected with MM-5TGM1 myeloma cells compared those in tumour-free control mice ( $119 \pm 5$ vs. $142 \pm 5 \mathrm{~g} / \mathrm{L}, \mathrm{n}=10$; Supplementary Figure $3 \mathrm{C}$. In this myeloma mouse model, we also did not find significant variation in either the proportion or absolute number of haematopoietic stem cells (LSK: $\left.\mathrm{Lin}^{-} \mathrm{c}-\mathrm{Kit}^{+} \mathrm{Sca}-1^{+}\right)(0.042 \% \pm 0.006 \%$ vs. $0.043 \% \pm 0.005 \%$; Fig. $2 \mathrm{E}, \mathrm{F})$. However, the frequency and absolute number of myeloid progenitor cells ( $\mathrm{LSK}^{-}: \mathrm{Lin}^{-} \mathrm{c}-\mathrm{Kit}^{+} \mathrm{Sca1}{ }^{-}$) in the bone marrow of MM-5TGM1 

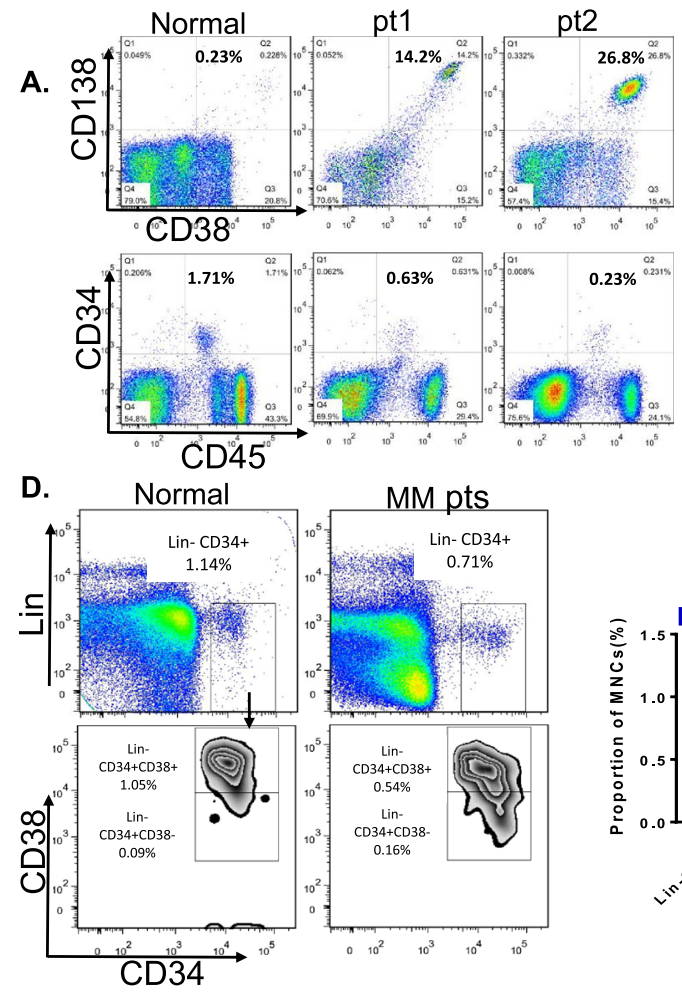

B.

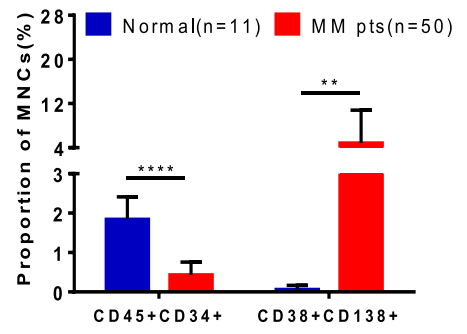

c.

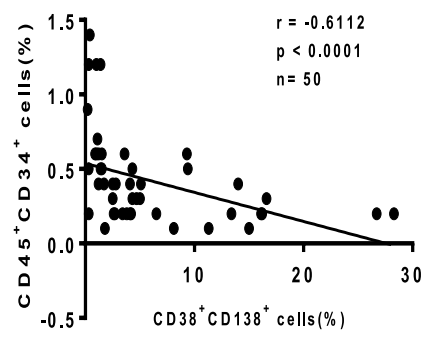

E.

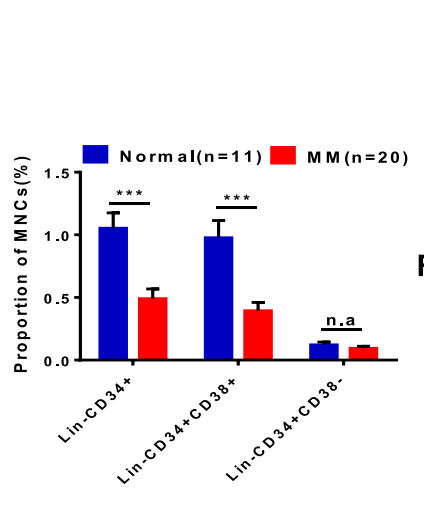

F.

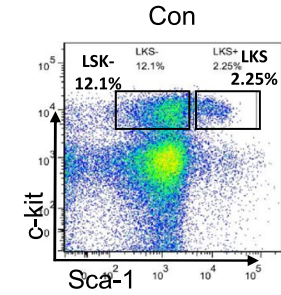

MM-5TGM1
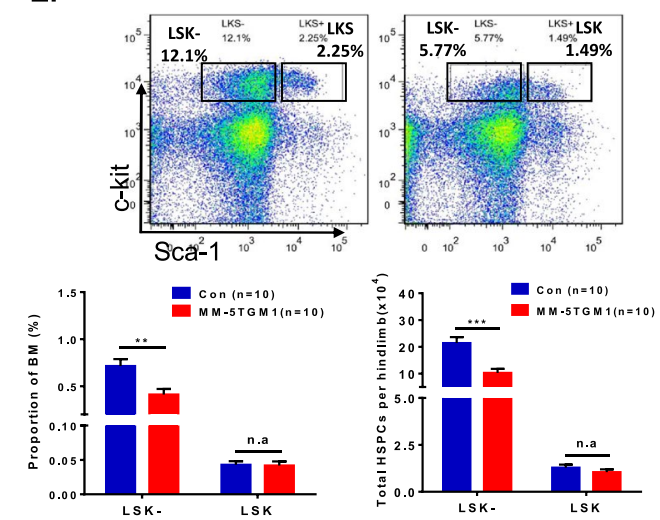

Figure 2. The proportion of HPCs is significantly decreased in the myeloma bone marrow in both primary patients and the MM mouse model. (A) Flow cytometry was used to detect CD138 ${ }^{+} \mathrm{CD} 38^{+}$myeloma cells and $\mathrm{CD} 45^{+} \mathrm{CD} 34^{+}$HSPCs in bone marrow samples from NDMM patients and normal donors. (B) Bar charts represent the mean proportions of $\mathrm{CD} 138^{+} \mathrm{CD} 38^{+}$plasma cells and $\mathrm{CD} 45^{+} \mathrm{CD} 34^{+} \mathrm{HSPCs}$ in normal donors and NDMM patients. (C) The proportion of $\mathrm{CD} 45^{+} \mathrm{CD} 34^{+}$cells in NDMM patients was negatively correlated with the proportion of $\mathrm{CD}_{138^{+}}$myeloma cells $(\mathrm{r}=-0.6112, p<0.0001, \mathrm{n}=50)$. (D) Flow plots (left) and a histogram (right) show the percentage of HSPCs in normal donors and NDMM patients. The subset composition of HSPCs $\left(\mathrm{Lin}^{-} \mathrm{CD} 34^{+}\right)$, including HPCs $\left(\mathrm{Lin}^{-} \mathrm{CD} 34^{+} \mathrm{CD} 38^{+}\right)$and HSCs $\left(\mathrm{Lin}^{-} \mathrm{CD} 34^{+} \mathrm{CD} 38^{-}\right)$, was determined in BM samples from normal donors and NDMM patients. (E) The subsets of HSPCs, including HPCs (Lin ${ }^{-}$, $\left.\mathrm{c}-\mathrm{kit}^{+}, \mathrm{Sca}-1^{-}\right)$and HSCs $\left(\mathrm{Lin}^{-}, \mathrm{c}-\mathrm{kit}^{+}, \mathrm{Sca}-1^{+}\right)$, were determined in BM samples from the 5TGM1 myeloma mouse model and control mice. (F) The proportion (left) and absolute number (right) of HSPC subsets were investigated ( $\mathrm{n}=10$ per group).

mice were significantly reduced compared to those in tumour-free control mice. In these two mouse groups, the frequency of $\mathrm{LSK}^{-}$cells was $(0.41 \% \pm 0.06 \%)$ versus $(0.71 \% \pm 0.07 \%), p=0.0052$, and the absolute number was $(10.27 \pm 1.50) \times 10^{4}$ versus $(21.43 \pm 2.23) \times 10^{4}, p=0.0006$, respectively. The results of the myeloma patient and mouse model experiments showed that myeloma cell infiltration mainly caused a decrease in HPCs in the bone marrow. These data also indicated that the differentiation of HSPCs was inhibited in the microenvironment of myeloma.

The differentiation of HSPCs is arrested in the HSC stage in the myeloma bone marrow. Longterm repopulating (LT) haematopoietic stem cells (HSCs) are the progenitor cells at the top of the haematopoietic hierarchy. Our data showed that the frequency of LT-HSCs ( Lin $\left.^{-}, \mathrm{c}_{-\mathrm{kit}}^{+}, \mathrm{Sca}-1^{+}, \mathrm{CD} 34^{-}, \mathrm{CD} 135^{-}\right)$in the bone marrow of the myeloma mouse model was notably increased compared to that in control mice $(21.13 \% \pm 0.85 \%$ vs. $7.55 \% \pm 1.46 \%, p<0.0001$; Fig. 3A,B). However, the frequencies of short-term HSCs (ST-HSCs: Lin', c-kit ${ }^{+}$, Sca- $\left.1^{+}, \mathrm{CD}_{4} 4^{+}, \mathrm{CD} 135^{-}\right)$and multipotent progenitor MPPs $\left(\mathrm{Lin}^{-}, \mathrm{c}-\mathrm{kit}^{+}, \mathrm{Sca}-1^{+}, \mathrm{CD} 34^{+}, \mathrm{CD} 135^{+}\right)$decreased significantly. Similarly, the absolute number of ST-HSCs and MPPs progressively decreased; by contrast, the absolute LT-HSC count showed no obvious variation. The results further suggested that the differentiation of LT-HSCs was defective in the bone marrow of myeloma mice.

Further analysis of the cell cycle and apoptosis showed that most HSPCs (LSK: $\mathrm{Lin}^{-} \mathrm{c}^{-} \mathrm{Kit}^{+} \mathrm{Sca}-1^{+}$, and $\mathrm{LSK}^{-}$: $\left.\mathrm{Lin}^{-} \mathrm{c}^{-} \mathrm{Kit}^{+} \mathrm{Sca}-1^{-}\right)$were arrested in G0 phase of the cell cycle in the myeloma model mice compared with tumourfree control mice (G0 phase, $\mathrm{LSK}^{-}: 41.88 \% \pm 6.97 \%$ vs. $18.48 \% \pm 1.56 \%, p=0.0083$; LSK: $55.60 \% \pm 2.61 \%$ vs. $18.85 \% \pm 1.01 \%, p<0.0001$; Fig. 3C). However, no obvious apoptosis was detected in HSPCs from myeloma mice (LSK ${ }^{-}: 8.24 \% \pm 1.48 \%$ vs. $7.30 \% \pm 0.85 \%$; LSK: $9.71 \% \pm 1.53 \%$ vs. $6.60 \% \pm 0.52 \%$; Fig. $3 \mathrm{D}$ ). Therefore, our results demonstrated that cell cycle arrest, but not apoptosis, likely affected the differentiation of HSPCs in response to the myeloma microenvironment. 
A.

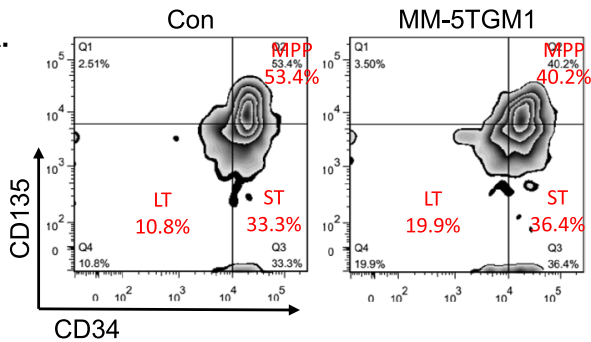

B.

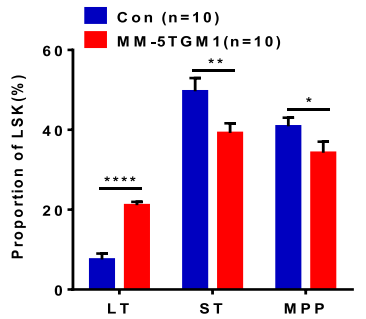

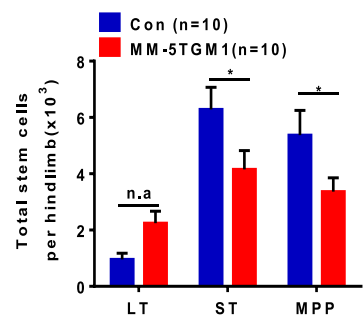

C.

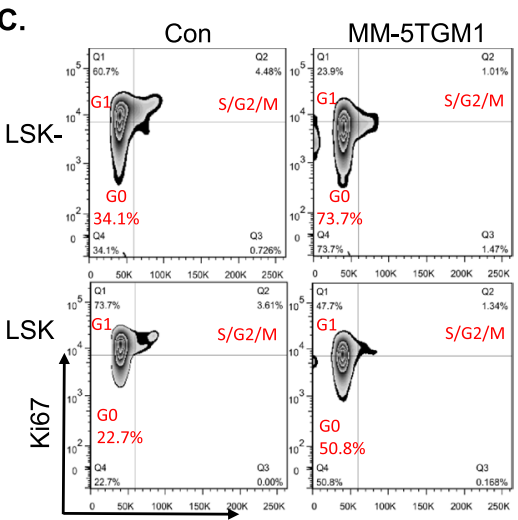

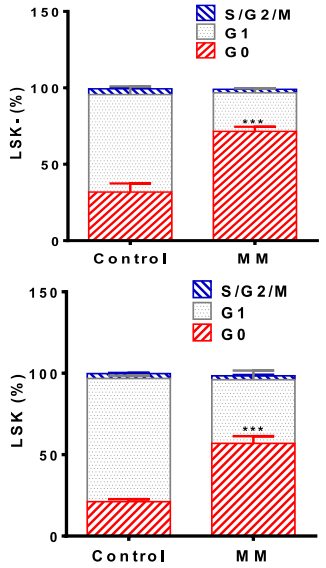

D.
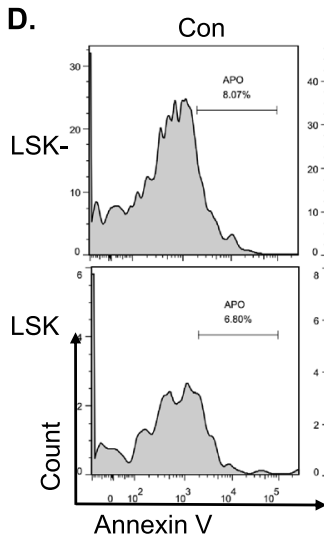

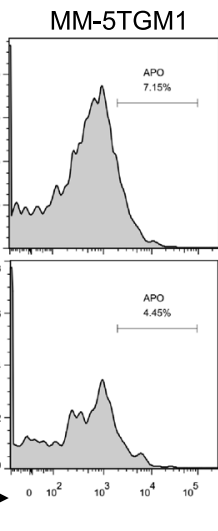

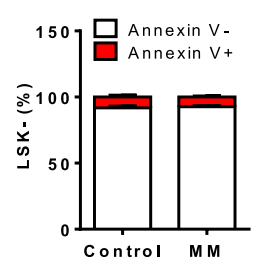

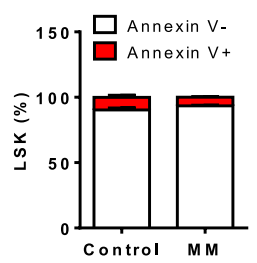

Hoechst33342

Figure 3. The differentiation of HSPCs arrested in the HSC stage in the myeloma bone marrow. (A) The subset of LSK cells, including LT-HSCs (Lin $\left.{ }^{-}, \mathrm{c}-\mathrm{kit}^{+}, \mathrm{Sca}-1^{+}, \mathrm{CD} 34^{-}, \mathrm{CD} 135^{-}\right)$, ST-HSCs $\left(\mathrm{Lin}^{-}, \mathrm{c}-\mathrm{kit}^{+}, \mathrm{Sca}-1^{+}, \mathrm{CD} 34^{+}\right.$, $\left.\mathrm{CD} 135^{-}\right)$, and MPPs $\left(\mathrm{Lin}^{-}, \mathrm{c}-\mathrm{kit}^{+}, \mathrm{Sca}-1^{+}, \mathrm{CD} 34^{+}, \mathrm{CD} 135^{+}\right)$, was determined in BM samples. (B) The proportions and absolute numbers of the LSK subset are shown. (C) Representative flow graphs (left) and histograms (right) show the cell cycle status of $\mathrm{LSK}^{-}\left(\mathrm{Lin}^{-} \mathrm{c}^{-} \mathrm{Kit}^{+} \mathrm{Sca}-1^{-}\right)$and LSK $\left(\mathrm{Lin}^{-} \mathrm{c}^{-} \mathrm{Kit}^{+} \mathrm{Sca}-1^{+}\right)$cells in BM from MM-5TGM1 mice and tumour-free control mice ( $\mathrm{n}=6$ in each group). (D) Apoptosis of LSK ${ }^{-}$and LSK cells from MM-5TGM1 mice and tumour-free control mice is shown ( $\mathrm{n}=6$ per group).

Erythrocyte differentiation of HSPCs is impaired in the myeloma microenvironment. Megakaryocytic-erythroid progenitors (MEPs) are considered to be the classical progenitors of functional red blood cells and platelets ${ }^{13}$. We analysed the quantity of common myeloid progenitors (CMPs) and their progeny, granulocyte-monocyte progenitors (GMPs) and megakaryocyte-erythrocyte progenitors (MEPs) in the bone marrow of

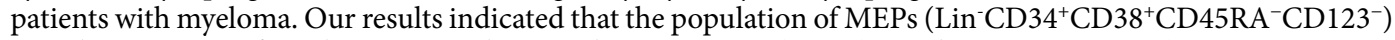
was the most significantly suppressed in myeloma patients $(\mathrm{n}=20)$, as shown in Fig. 4 A $(3.52 \% \pm 0.35 \%$ vs. $8.43 \% \pm 0.89 \%, p<0.0001)$. Furthermore, a colony formation assay of CD $34^{+}$HSPCs from myeloma patients or healthy control subjects was performed. The data indicated that colony formation, especially BFU-E colony formation, was significantly defective in myeloma CD34 ${ }^{+}$HSPCs compared to normal control CD34 $4^{+} \mathrm{HSPCs}$ $(31.00 \pm 3.98$ vs. $44.40 \pm 3.14, p<0.01$, Fig. $4 \mathrm{~B})$. Experiments in the myeloma mouse model confirmed these results. The proportion of MEPs was significantly decreased in MM-5TGM1 mice compared to tumour-free control mice $(12.10 \% \pm 0.87 \%$ vs. $23.85 \% \pm 1.59 \%, p<0.0001$, Fig. $4 \mathrm{C})$. Moreover, the quantities of the proerythroblast (ProEs: CD71 ${ }^{+}$Ter119 $9^{\text {low }}$ ) and Ter119+ erythroblast subpopulations (Ery.A, Ery.B and Ery.C) were notably reduced in MM-5TGM1 mice (ProEs: $(0.78 \pm 0.10) \times 10^{5}$ vs. $(3.28 \pm 0.38) \times 10^{5}, p<0.0001$; Ter $119^{+}$: $(54.10 \pm 4.25) \times 10^{5}$ vs. $(98.25 \pm 9.20) \times 10^{5}, p<0.001$; Fig. $\left.4 \mathrm{D}-\mathrm{F}\right)$. However, the proportion of Ter $119^{+} 71^{-}($Ery.C) increased notably. In order to confirm this result, we further analyzed the bone marrow smears of myeloma patients $(n=10)$ and healthy donors $(n=4)$. We found that the proportion of erythroblasts decreased in bone marrow of MM patients (supplementary Figure 4A), but the proportion of late erythroblast cells increased in erythroblasts subset (supplementary Figure 4B).

RNA sequencing analysis indicates the downregulation of GATA1 and KLF1 and upregulation of CCL3 in CD34+ cells from myeloma patients. To clarify the signalling pathway involved in the defective erythropoiesis of HSPCs within the bone marrow of myeloma patients, RNA sequencing was performed. A total of 1243 differentially expressed genes (DEGs) were identified in CD34 ${ }^{+}$cells between myeloma patients $(n=8)$ and healthy donors $(n=3), 852$ of which were upregulated and 391 of which were downregulated (FC $>2.0, p<0.01$; Fig. 5A). Gene set enrichment analysis ${ }^{14}$ revealed that the gene sets related to erythrocyte development and erythrocyte differentiation were significantly suppressed in CD $34^{+}$cells from MM patients but enriched in those from normal control subjects (Fig. 5B). More importantly, the expression of transcription factors, such as GATA1 and KLF1, which govern erythrocyte differentiation, was significantly downregulated in $\mathrm{CD} 34^{+}$HSPCs from myeloma patients. Meanwhile, the expression of genes involved in the chemokine signalling pathway, including CCL3, CCL4, CCL5 and CCL20, was upregulated (Fig. 5C-F). This result supports the 
A.
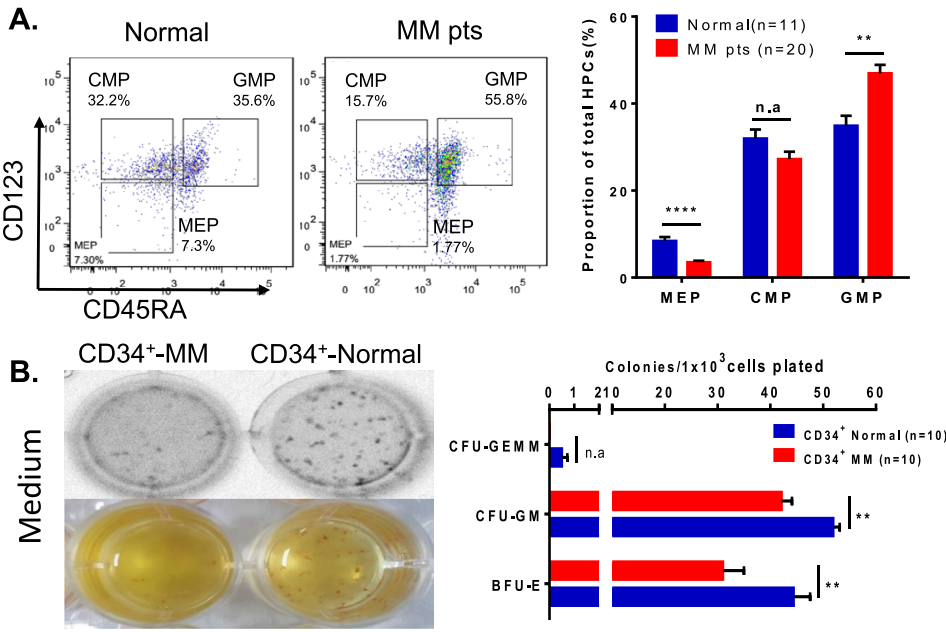

C.
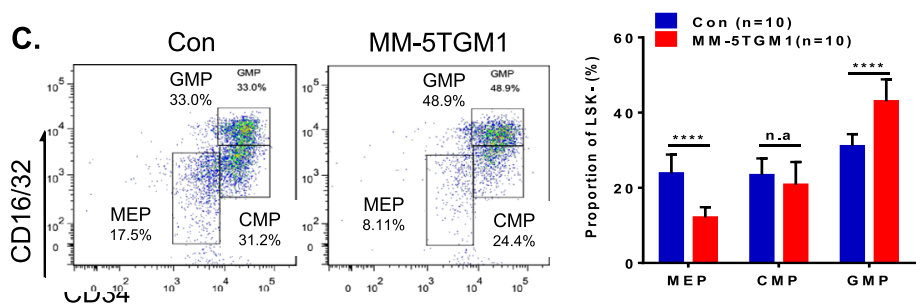

D.

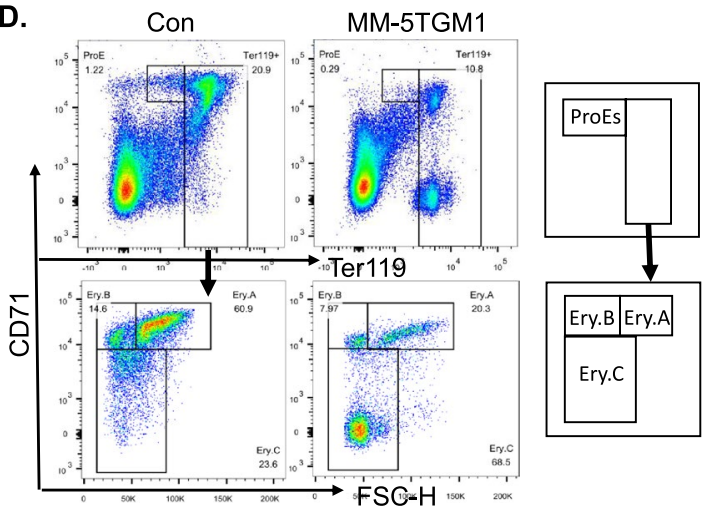

E.

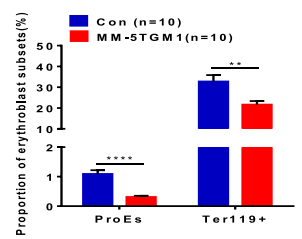

F.

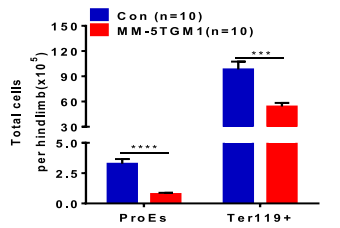

Figure 4. Erythroid differentiation of HSPCs was inhibited under the bone marrow microenvironment of MM patients and the 5TGM1 MM mouse model. (A) Flow plots (left) and a histogram (right) show the percentage of HSPCs in normal donors $(n=11)$ and NDMM patients $(n=20)$. The subset composition of HSPCs, including $\mathrm{CMP}\left(\mathrm{Lin}^{-}, \mathrm{CD}^{4} 4^{+}, \mathrm{CD} 38^{+}, \mathrm{CD} 123^{\mathrm{lo}}, \mathrm{CD}_{5} 5 \mathrm{RA}^{-}\right), \mathrm{GMP}\left(\mathrm{Lin}^{-}, \mathrm{CD} 34^{+}, \mathrm{CD} 38^{+}, \mathrm{CD} 123^{\mathrm{lo}}, \mathrm{CD} 45 \mathrm{RA}^{+}\right)$, and MEP

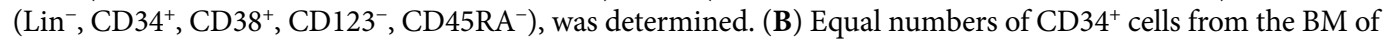
normal donors and NDMM patients were seeded in semisolid methylcellulose complete medium. The numbers of BFU-E, CFU-GM and CFU-GEMM colonies were calculated after 14 days of co-culture (upper). The means of the absolute numbers of colonies are shown (lower, $\mathrm{n}=10$ per group). (C) The subset composition of HSPCs, including CMP $\left(\mathrm{Lin}^{-}, \mathrm{c}-\mathrm{kit}^{+}, \mathrm{Sca}-1^{-}, \mathrm{CD} 34^{+}, \mathrm{CD} 16 / 32^{\mathrm{lo}}\right), \mathrm{GMP}\left(\mathrm{Lin}^{-}, \mathrm{c}-\mathrm{kit}^{+}, \mathrm{Sca}-1^{-}, \mathrm{CD} 34^{+}, \mathrm{CD} 16 / 32^{\mathrm{hi}}\right)$, and MEP (Lin ${ }^{-}, \mathrm{c}^{-\mathrm{kit}^{+}}, \mathrm{Sca}-1^{-}, \mathrm{CD} 34^{-}, \mathrm{CD} 16 / 32^{-}$), was determined in BM samples from MM-5TGM1 mice and control mice. (D) Erythroblast subsets were analysed by flow cytometry based on the expression of CD71 and Ter-119 as follows: ProE (CD71 $1^{+}$Ter-119 $\left.{ }^{\text {low }}\right)$, Ery.A, Ery.B, and Ery.C (Ter-119 $)$. (E) The proportion of different erythroblasts in BM from MM-5TGM1 mice and tumour-free control mice. (F) Absolute number of ProEs, Ter$119^{+}$, Ery.A, Ery.B and Ery.C in the BM of MM-5TGM1 mice and tumour-free control mice.

notion that cytokines or chemokines in the myeloma microenvironment play a pivotal role in the suppression of HSPC differentiation through the downregulation of transcription factor expression.

High levels of CCL3 in myeloma bone marrow plasma cells inhibits erythrocyte differentiation. In addition to the intrinsic deficits in the erythropoiesis of CD34 ${ }^{+}$HSPCs with downregulated GATA1 expression in a myeloma bone marrow microenvironment, we found that the bone marrow plasma cells of myeloma patients further suppressed the BFU-E formation of $\mathrm{CD} 34^{+}$cells both from myeloma patients and healthy donors (Fig. 6A). Our previous clinical analysis showed in Table 1 that bone disease was correlated with anaemia in myeloma patients. CCL3 is one of the major cytokines secreted by MM cells that promotes tumour cell proliferation and disrupts normal haematopoietic niches, thus inducing myeloma-related bone disease ${ }^{15,16}$. Our RNA-seq data indicated that the expression levels of the chemokines CCL3 and CCL20, which play an important role in the pathogenesis of myeloma-induced bone disease ${ }^{17}$, were upregulated in $\mathrm{CD} 34^{+}$cells in bone marrow from myeloma patients. Further ELISA analysis showed that the secreted level of CCL3 significantly increased in the bone marrow plasma cells of myeloma patients and in the MM-5TGM1 mouse model (human plasma: $779.2 \pm 28.97 \mathrm{pg} / \mathrm{mL}$ vs. $83.52 \pm 5.31 \mathrm{pg} / \mathrm{mL}, p<0.0001$; mouse serum: $13.03 \pm 1.94 \mathrm{pg} / \mathrm{mL}$ vs. $5.33 \pm 1.46 \mathrm{pg} / \mathrm{mL}$, $p=0.0317$; mouse plasma: $34.03 \pm 1.32 \mathrm{pg} / \mathrm{mL}$ vs. $15.86 \pm 3.88 \mathrm{pg} / \mathrm{mL}, p=0.0114$; Fig. $6 \mathrm{~B}, \mathrm{C})$. The Kaplan-Meier analysis based on the GEO data of individuals with NDMM (GSE2658) indicated that a high level of CCL3 significantly correlated with shorter overall survival in patients with myeloma (56.05 months vs. unreached, $\mathrm{HR}=1.943, p=0.0034$; Fig. $6 \mathrm{D}$ ). In particular, the clinical characteristics of patients with anaemia indicated that the Hgb level was negatively correlated with bone disease. The patients with higher $\mathrm{Hgb}[102.9 \pm 3.72 \mathrm{~g} / \mathrm{L}$ $(\mathrm{n}=47)]$ had mild bone disease $(0-2$ stage), and the patients with advanced anaemia [86.38 $\pm 2.13 \mathrm{~g} / \mathrm{L}(\mathrm{n}=102)]$ had severe bone disease ( $3-4$ stage) $(p<0.0001$, Fig. $6 \mathrm{E})$. These results further support the notion that soluble CCL3 in the myeloma microenvironment is involved in the process of myeloma-related anaemia. 


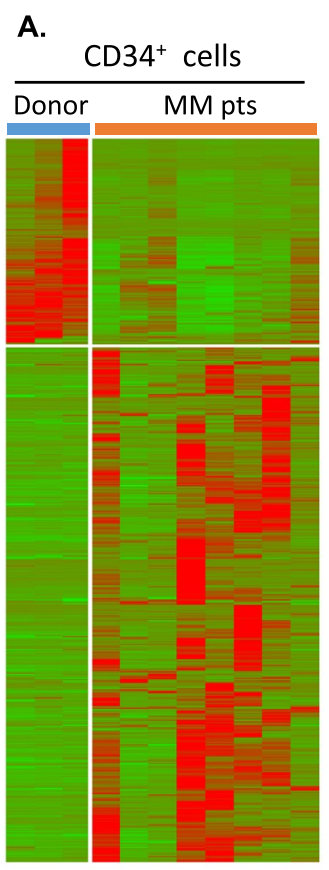

B.

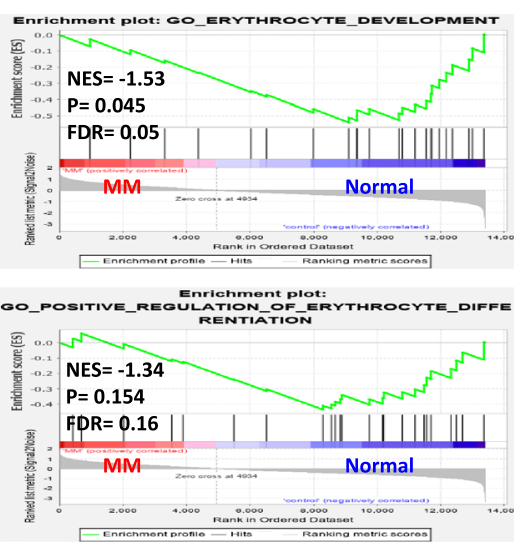

c.

E.

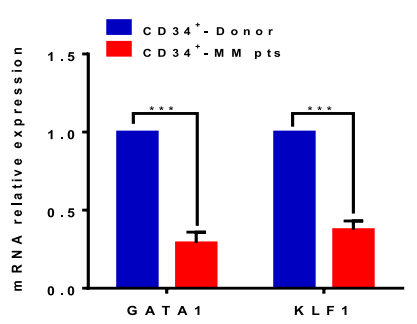

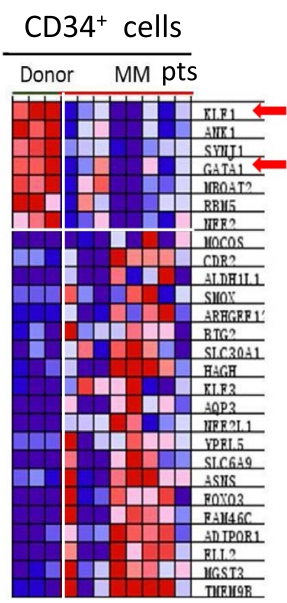

F.

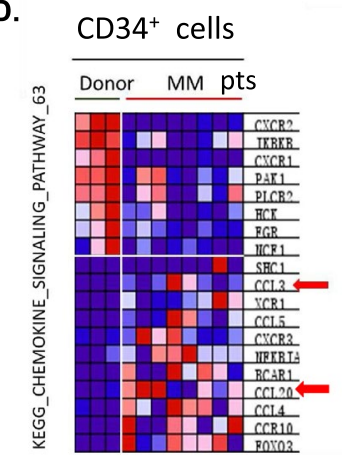

Figure 5. RNA-seq identified the downregulation of transcription factors involved in erythroid differentiation of HSPCs in the myeloma microenvironment. (A) Heatmap of differentially expressed genes in CD $34^{+}$cells from MM patients and healthy controls. (B) GSEA comparison of gene expression inCD $34^{+}$cells from MM patients and normal controls with respect to erythrocyte development (upper panel) and positive regulation of erythrocyte differentiation (lower panel). The normalized enrichment scores (NESs), $p$ values and FDR q-values are indicated in each plot. (C) GSEA revealed that the expression of the transcription factors GATA1 and KLF1 was significantly decreased in CD34 $4^{+}$cells from MM patients. (D) KEGG (Kyoto Encyclopedia of Genes and Genomes) pathway enrichment analysis of the genes involved in the chemokine signalling pathway. CCL3 was upregulated in CD34 ${ }^{+}$cells from myeloma patients. (E) RT-PCR indicated that the expression of the transcription factors GATA1 and KLF1 was significantly decreased in $\mathrm{CD} 34^{+}$cells from MM patients. (F) RT-PCR indicated that the expression of CCL3 was significantly increased in CD34+ cells from MM patients.

Activation of the CCL3/CCR1/p38 signalling pathway disrupts erythroid differentiation. Our previous data indicated that the high level of CCL3 in the myeloma bone marrow microenvironment is probably involved in the myeloma-mediated inhibition of erythropoiesis. Therefore, the expression of CCL3 receptors (CCR1, CCR4 and CCR5) on the surface of CD34 $4^{+}$cells was detected by flow cytometry. The data showed that the levels of CCR1 and CCR5, but not CCR4, were significantly increased on the HSPCs of myeloma patients compared to healthy donors (CCR1: $5.13 \% \pm 1.32 \%$ vs. $0.85 \% \pm 0.24 \%, p=0.0096$; CCR5: $5.72 \% \pm 1.57 \%$ vs. $1.91 \% \pm 0.37 \%, p=0.0199$; Fig. 7A). Moreover, antagonists of CCR1 (BX471), CCR4 (AZD2098) and CCR5 (Maraviroc) were used to inhibit CCL3 activity in the co-culture system of CD34 ${ }^{+}$cells and bone marrow plasma cells from patients. As shown in Fig. 7B, we found that blocking CCL3/CCR1 signalling with BX471 efficiently restored BFU-E formation. However, the CCR4 antagonist (AZD2098) and CCR5 antagonist (Maraviroc) had limited effects on rescuing BFU-E formation. As a downstream effector of CCL3/CCR1 signalling, phosphorylated p38 in HSPCs treated with CCL3 and the CCR1 antagonist BX471 was further analysed. Immunofluorescence staining indicated that CCL3 effectively activates p 38 signalling with high levels of phosphorylated p 38 in $\mathrm{CD} 34^{+}$cells derived from myeloma patients. Importantly, blocking the CCL3 signal with BX471 (an antagonist of CCR1) effectively restored the effect of CCL3 on the downregulation of the transcription factor GATA1 in $\mathrm{CD}^{+}{ }^{+}$cells in the myeloma microenvironment (Fig. 7C). Real-time PCR and Western blotting further indicated the recovery of GATA1 expression at both the mRNA and protein levels after blocking the CCL3/CCR1/phosp38 signalling pathway in $\mathrm{CD} 34^{+}$cells from myeloma patients (Fig. 7D,E). Compared to the $\mathrm{CD} 34^{+}$cells from myeloma bone marrow, the expression of GATA1 in normal CD34 ${ }^{+}$slightly suppressed by the CCL3 treatment which also caused by pho-p38 (supplementary Figure 5). Taken together, the results demonstrated that a high level of CCL3 activates p38 signalling, resulting in the suppression of transcription factor GATA1 expression and blocking erythroid differentiation of $\mathrm{CD} 34^{+} \mathrm{HSPCs}$ in the MM bone marrow microenvironment.

\section{Discussion}

Anaemia is the most common complication of many kinds of cancer, including MM, and is associated with worse clinical outcomes, including longer length of hospital stay, diminished quality of life, and increased morbidity and mortality ${ }^{18,19}$. The most frequent underlying pathophysiological mechanisms reported previously in 
A.

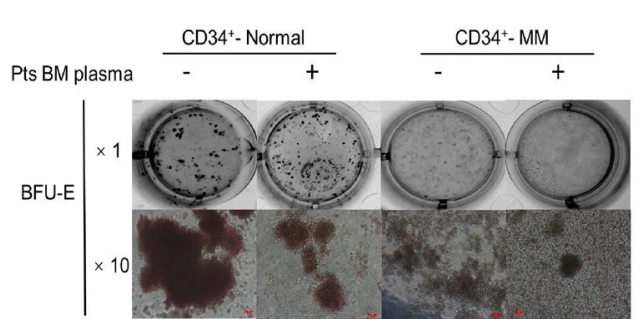

C.

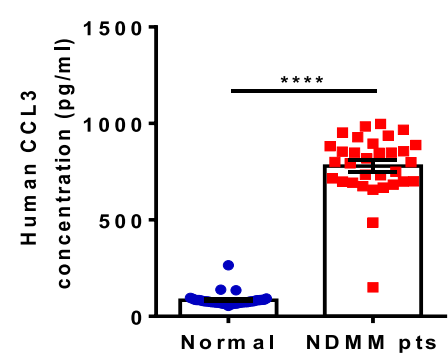

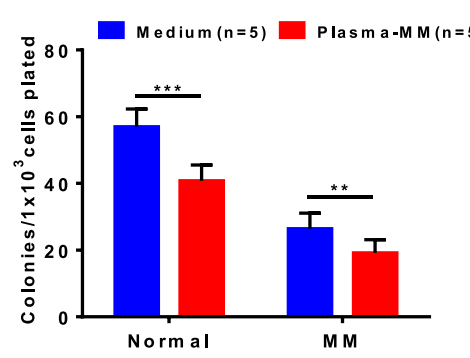

D.

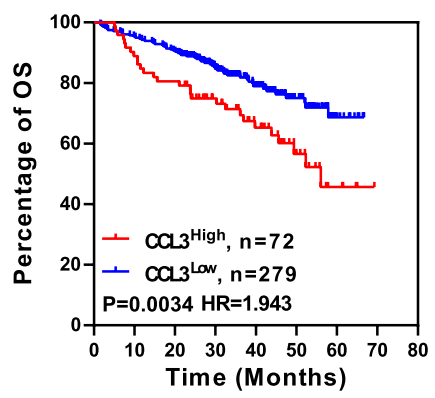

B.

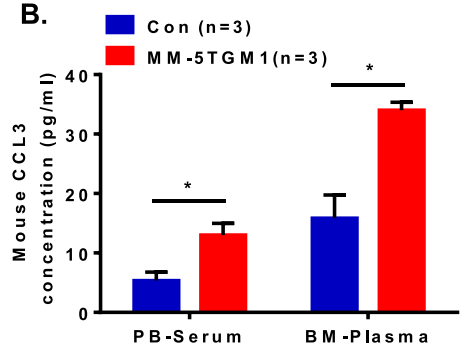

E.

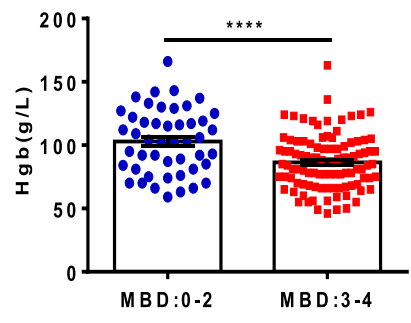

Figure 6. Bone marrow plasma inhibited erythroid colony formation of HSPCs by increasing CCL3 levels in the MM microenvironment. (A) A CFC assay was utilized to quantify the BFU-E colony formation of CD34 ${ }^{+}$ cells after co-culture with or without BM plasma from MM patients for 14 days. The absolute numbers of BFU-E colonies are shown on the right. (B) The concentrations of CCL3 in the bone marrow plasma cells and PB serum of 5TGM1 MM mice and control mice were detected with ELISA. (C) The concentration of CCL3 in the bone marrow plasma cells of MM patients was determined with ELISA (normal, $\mathrm{n}=40$; NDMM pts, $\mathrm{n}=32$ ). (D) Kaplan-Meier analysis showed shorter overall survival in MM patients with high levels of CCL3 based on the GEO dataset GSE2658. (E) The clinical data indicated that the more severe the bone disease is (MBD 3-4), the greater the decrease in haemoglobin in MM patients (MBD: 0-2, $n=47 ;$ MBD: 3-4, $n=102$ ).

tumour-induced anaemia include aberrant iron metabolism, renal impairment, anaemia of chronic disease and a hypoxic microenvironment ${ }^{20,21}$. Although marrow replacement by malignant plasma cell infiltration is widely considered the basis for myeloma-related anaemia ${ }^{22}$, the molecular mechanism has not been fully clarified.

In this study, the data of our large cohort of 1363 newly diagnosed myeloma patients demonstrated that $84.4 \%$ of patients had anaemia regardless of sex $(\mathrm{Hgb}<120 \mathrm{~g} / \mathrm{L})$, and more than $50 \%(753 / 1363)$ of patients had moderate $(\mathrm{Hgb}=90-120 \mathrm{~g} / \mathrm{L})$ or severe anaemia $(\mathrm{Hgb}=60-90 \mathrm{~g} / \mathrm{L})$ at the time of diagnosis. Anaemia was positively correlated with myeloma cell infiltration in the bone marrow of the patients and worsened with the progression of the disease. The patients with anaemia had significantly shorter progression-free survival and overall survival (medium PFS, 19.7 months vs. 28.5 months, $p<0.0001$; medium OS, 29.0 months vs 48.0 months, $p<0.0001$; Hgb cut-off $90 \mathrm{~g} / \mathrm{L}$ ).

Bone marrow is the main location of the growth and differentiation of hematopoietic stem cells (HSPCs). The interactions of HSPCs with other cells, secreted and membrane-bound proteins and extracellular matrix components in the bone marrow regulate HSPC differentiation ${ }^{12,23}$. Previously, Ingmar Bruns and colleagues reported that HSPCs were suppressed under a myeloma microenvironment ${ }^{24}$. Our results further demonstrated that the number of HPCs $\left(\mathrm{Lin}^{-} \mathrm{CD} 34^{+} \mathrm{CD} 38^{+}\right)$, but not $\mathrm{HSCs}\left(\mathrm{Lin}^{-} \mathrm{CD} 34^{+} \mathrm{CD} 38^{-}\right)$, was more significantly decreased in the bone marrow of myeloma and negatively correlated with the quantity of $\mathrm{CD} 38^{+} \mathrm{CD} 138^{+}$tumour cells $(\mathrm{r}=-0.6112)$. This result was confirmed in a myeloma mouse model. In particular, the LSK, short-term LSK and MPP populations, but not the long-term LSK population, were decreased in the bone marrow of myeloma mice. These data suggested that the differentiation of HPCs from HSCs was blocked under the microenvironment of myeloma. We also found that HSPCs were arrested in G0 phase and that apoptosis was not significantly changed in the bone marrow microenvironment of myeloma. Flow cytometry data showed that the population of MEPs, which differentiated from HPCs and are precursors of erythrocytes, was decreased both in the bone marrow of myeloma patients and in the MM mouse model (MM-5TGM1). We found that the frequencies of the ProEs and the A and B erythroblasts (Ery.A and Ery.B) subpopulations were reduced while that of the C erythroblasts (Ery.C) were increased in myeloma mice (Fig. 4D,E). This result was consistent with the data of MM patients shown in supplementary Figure 4. These results from patients and mouse model suggested that the differentiation of erythropoiesis was defected under the microenvironment of myeloma. However, we did not find the notable changes of Annexin $\mathrm{V}^{+}$apoptosis cells and the cell cycle arrest in Ery.C (data not shown). Moreover, due to the infiltration of myeloma cells, macrophage function was abnormal ${ }^{2,25}$. We speculate that enucleation and differentiation of Ery.C cells were impacted under the microenvironment of myeloma with the macrophage disfunction. This would be involved in the mechanisms for the Ery.C cell accumulation. This hypothesis needs to be further investigated in the future. Our data also showed that the erythrocyte differentiation (BFU-E and 
A.

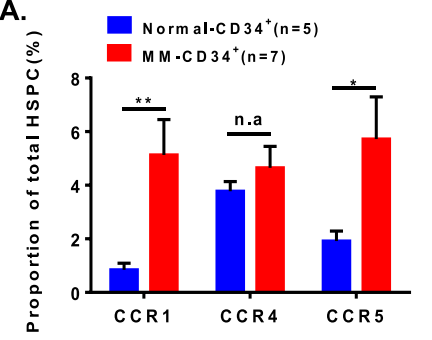

B.

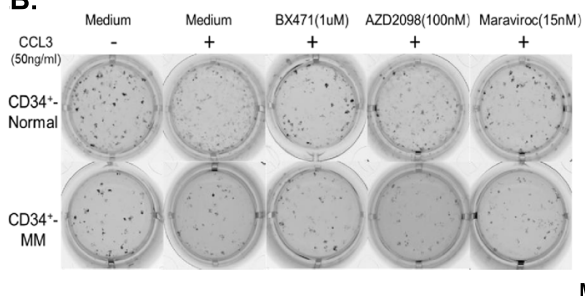

BFU-E colonies $/ 1 \times 10^{3}$ cells plated

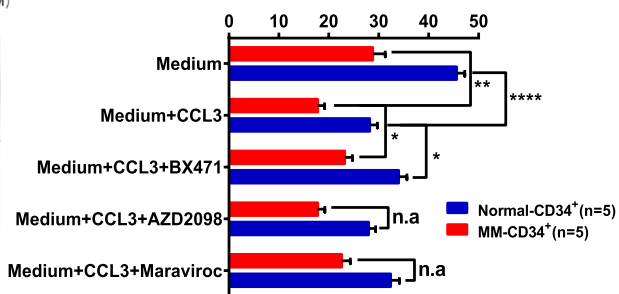

c.

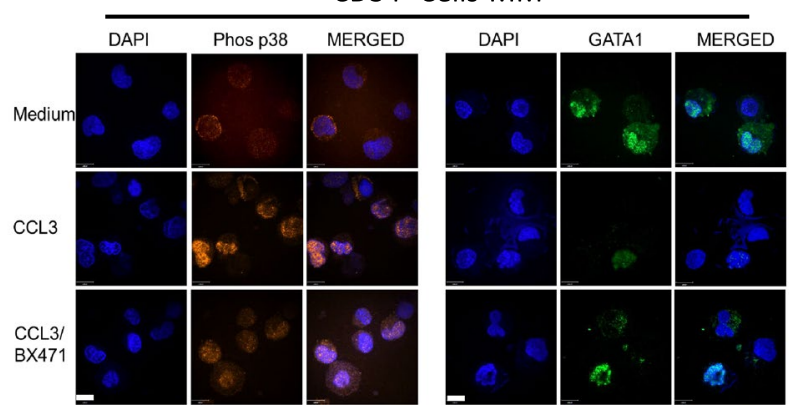

D.

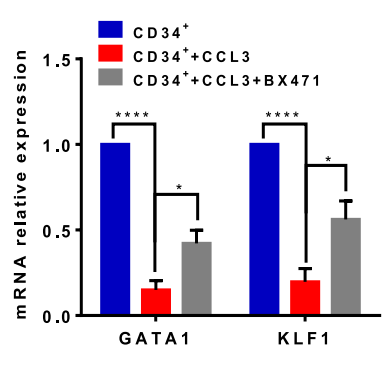

E.

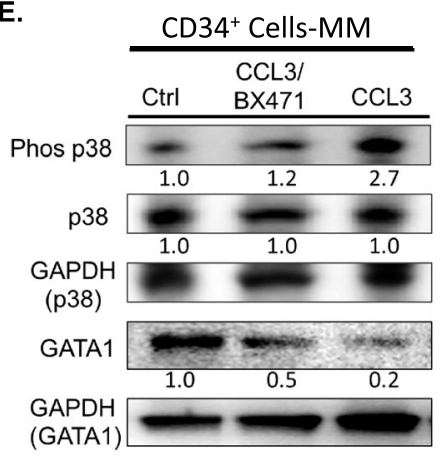

Figure 7. A high level of CCL3 suppresses erythroid differentiation of HSPCs via activation of the CCL3/ CCR1/p38 signalling pathway in MM. (A) Flow cytometry analysis showing the expression of CCL3 receptors (CCR1, CCR4 and CCR5) in CD34 cells from normal donors and NDMM patients. (B) The CFC assay showed the BFU-E colony-forming ability of CD34+ cells in the presence or absence of CCL3 $(50 \mathrm{ng} / \mathrm{ml})$ and the CCR1 antagonist BX471 $(1 \mu \mathrm{M})$, the CCR4 antagonist AZD2098 (100 nM), or the CCR5 antagonist maraviroc $(15 \mathrm{nM})$ (left graphs). The histograms indicate the absolute number of BFU-E colonies formed (right). (C) Immunofluorescence staining of phosphorylated p38 (orange) and GATA1 (green) in EPOinduced HSPC $\left(\mathrm{CD} 34^{+}\right)$differentiation in the presence or absence of CCL3/BX471. Nuclei were stained with DAPI. Fluorescence images viewed under a confocal microscope were acquired. The scale bar indicates $10 \mu \mathrm{m}$. (D) RT-PCR analysis showed the expression levels of GATA1 and KLF1 in CD34+ cells in the presence or absence of CCL3/BX471. GAPDH expression was used as an internal control. (E) Western blotting analysis of phosphorylated p38 (phos-p38), total p38 and GATA1 after the induction of erythroid differentiation in the presence or absence of CCL3/BX471. GAPDH expression was used as an internal control.

CFU-GM) was further inhibited in CD34+ cells derived from MM patients compared with those derived from healthy donors. Therefore, these results supported the halted differentiation of HSPCs developmentally arrested in the HSC stage that is caused by MM cell infiltration.

To further clarify the molecular mechanism of the defective differentiation of HSPCs in the bone marrow of myeloma, high-throughput RNA-seq was utilized to determine the differential gene expression in CD34 ${ }^{+}$cells between MM patients and healthy donors. There were 1243 DEGs, of which 852 were upregulated and 391 were downregulated, in $\mathrm{CD}_{3} 4^{+}$cells of MM patients compared to healthy controls $(p<0.01)$. In particular, the expression of the master regulators of erythropoiesis ${ }^{26-28}$, including GATA1 and KLF1, was obviously decreased in myeloma CD34 ${ }^{+}$cells $(\mathrm{FC}<-2.0, p<0.01)$. However, the expression of chemokine genes, such as CCL3 and CCL20, was significantly upregulated. The results suggested that the gene expression profile of HSPCs varied inherently under the bone marrow microenvironment of myeloma, which would disrupt erythroid differentiation.

In addition, it has been well established that malignant plasma cell infiltration disrupts bone marrow homeostasis between the highly organized cellular and extracellular compartments ${ }^{9}$. The release of cytokines and growth factors from malignant cells and nonneoplastic bystander cells leads to alterations in the bone marrow cytokine milieu, which interferes with the behaviour of $\mathrm{HSPCs}^{29}$. Our study showed that the BFU-E formation of HSPCs was further diminished when they were co-cultured with the bone marrow plasma cells of myeloma patients. Interestingly, our clinical data clearly showed that anaemia was positively correlated with bone disease severity in patients with myeloma. Patients with more severe bone lytic lesions would concurrently have more severe anaemia. These results implied that the occurrence of anaemia was highly associated with bone disease. There are some common mechanisms involved in osteoclast differentiation and erythropoiesis induced by myeloma cell infiltration. Fields SZ et al. reported that dysregulated signalling by activin receptor pathways is implicated in certain instances of anaemia, myeloma-associated osteolysis, and metastatic bone disease, as well as in carcinogenesis ${ }^{30}$. Deshet-Unger $\mathrm{N}$ and colleagues recently reported that erythropoietin (EPO), the cytokine required for erythropoiesis, directly stimulates osteoclast precursors and induces bone loss in murine multiple myeloma $^{31,32}$. EPO targets the monocytic lineage by increasing the number of bone monocytes/macrophages, preosteoclasts, and mature osteoclasts. Their study also suggested the potential mechanism involved in both 
erythropoiesis and bone loss. However, the research of Bordini et al. ${ }^{25}$ excluded the possibility that anaemia results from defective erythropoietin production, inflammation or increased hepcidin expression in myeloma.

Cytokines and chemokines are the main components of bone marrow plasma cells and play a prominent role in the pathogenesis of myeloma-related bone disease. CCL20 is a chemokine involved in the Th17 pathway and has also been implicated in MM osteolytic disease ${ }^{33}$. The chemokine cytokine ligand 3 (CCL3/MIP-1 $\alpha$ ) is a pro-inflammatory protein and chemokine mainly secreted by myeloma plasma cells and contributes to the development of myeloma-induced bone disease by supporting tumour growth and regulating osteoclast (OC) differentiation. CCL3 also reduces bone formation by inhibiting osteoblast (OB) function and therefore contributes to $\mathrm{OB} / \mathrm{OC}$ uncoupling in $\mathrm{MM}^{15,16}$. Currently, the role of CCL3 in the erythropoietic lineage is not fully understood. Chen et al. and Lentzsch et al. reported that the interaction of CCL3 and CCR1 effectively triggered the activation of MAPK and AKT pathways, which are involved in cell proliferation and differentiation ${ }^{34,35}$. A recent study from our colleagues Wang et al. ${ }^{36}$ showed that the increase in CCL3 levels in AML bone marrow partially causes defects in erythropoiesis and results in leukaemia-related anaemia. CCL3 elevation suppressed erythropoiesis in the bone marrow of AML via CCL3/CCR1/phos-p38 activation. Perturbations in the bone marrow microenvironment are increasingly recognized to have key roles in initiating and supporting oncogenesis in both AML and $\mathrm{MM}^{37}$. Increased CCL3 expression has also been reported in some bone marrow failure syndromes, such as aplastic anaemia ${ }^{38}$. Based on previous reports, we first analysed our clinical data. We found that the level of CCL3 was significantly elevated in the BM plasma cells of MM. Consistent with Lentzsch et al. ${ }^{35}$, myeloma patients with high levels of CCL3 had shorter survival (Fig. 6). Moreover, the expression of the CCL3 receptors CCR1 and CCR5 was significantly increased on the surface of HSPCs from myeloma patients. CCR1 and CCR5 are the key players involved in the localization and growth of myeloma cells and in the development of osteolytic lesions ${ }^{39,40}$. Therefore, the increased levels of CCR1 and CCR5 expression on HSPCs indicated that myeloma cells adhere to HSPCs and promote myeloma cell localization to and proliferation in bone marrow. However, this speculation needs to be further investigated in the future. Our data also showed that increasing CCL3 levels effectively inhibited the BFU-E formation of CD34 ${ }^{+}$HSPCs.

The MAPK signalling pathway, which includes ERK, p38 and JNK, is an important downstream modulator of CCL3 and plays a pivotal role in myeloma cell proliferation and myeloma-mediated bone disease ${ }^{41}$. Hernandez et al. reported that acetylation and MAPK phosphorylation cooperate to regulate the degradation of active GATA- $1^{42}$. Therefore, we investigated the effect of CCL3/phos-p38 activation on the erythropoiesis of HSPCs in the myeloma microenvironment. Interestingly, we found that a high level of CCL3 significantly suppressed GATA1 expression at both the mRNA and protein levels in $\mathrm{CD} 34^{+}$cells in myeloma bone marrow. Our co-culture and rescue studies showed that a high level of CCL3 promoted the phosphorylation of $\mathrm{p} 38$ and suppressed the transcription and protein expression of GATA1. Inhibition of CCL3 activity with an antagonist of CCR1 (BX471) effectively restored the expression of GATA1 via inhibition of p38 phosphorylation and rescued BFU-E colony formation. Altogether, the data from our study showed that CCL3/CCR1/phos-p38 plays a pivotal role in erythropoiesis of myeloma HSPCs via downregulation of the transcription factor GATA1.

In summary, our study demonstrated that the MM microenvironment suppresses the erythroid differentiation capability of HSPCs and resulted in myeloma-related anaemia. Elevated CCL3 levels repress the erythroid differentiation of HSPCs in the bone marrow niche by suppressing the expression of the core erythroid transcription factors GATA1 and KLF1, causing insufficient erythropoiesis, as shown in our working model (Supplementary Figure 6). CCL3 is an important chemokine in myeloma pathogenesis that not only participates in inflammatory reactions and bone disease ${ }^{40}$ and is an adjuvant for immunotherapy ${ }^{43,44}$ but also plays a suppressive role in erythropoiesis and anaemia, as we reported here. Therefore, our study strongly supports the rationale for the development of novel agents that simultaneously suppress myeloma cell growth and myeloma-related bone disease and compensate for normal haematopoiesis to prevent the activation of CCL3 and consequently improve myeloma-related anaemia.

\section{Conclusions}

Our results demonstrated that myeloma cells secreted high levels of CCL3 in the bone marrow microenvironment and caused defective erythropoiesis of HSPCs by downregulating the expression of transcription factors in HSPCs, which resulted in anaemia. Targeting CCL3 would be a potential treatment strategy against bone disease and anaemia, which could essentially "kill two birds with one stone" in patients with multiple myeloma.

\section{Methods}

Clinical data analysis. A total of 1363 newly diagnosed MM patients who visited our centre from 1997 to 2017 were enrolled in this analysis. The clinical features are described in Table 1. Multiple myeloma and related anaemia were diagnosed according to the criteria of the International Myeloma Working Group and the World Health Organization ${ }^{45,46}$.

Patient samples. Bone marrow specimens from 50 newly diagnosed MM patients and 11 healthy control subjects were used in the in vitro study. Table 2 shows the detailed characteristics of the patients. Bone marrow biopsies were obtained following the protocol of the Department of Lymphoma and Myeloma, Institute of Hematology \& Blood Disease Hospital, Chinese Academy of Medical Sciences and Peking Union Medical College (Tianjin, China). All subjects involved in the study provided written informed consent according to the Declaration of Helsinki; this research was approved by the Medical Ethics Committee of the Chinese Academy of Medical Sciences, Institute of Hematology and Blood Diseases Hospital Ethics Board (KT2019022-EC-1, 201903-08). 


\begin{tabular}{|c|c|c|c|c|}
\hline Characteristics & All patients (\%) & $\mathrm{Hgb}>90 \mathrm{~g} / \mathrm{L}(\%)$ & $\mathrm{Hgb} \leq 90 \mathrm{~g} / \mathrm{L}(\%)$ & $p$ \\
\hline No. of patients & 50 & $24(48.0 \%)$ & $26(52.0 \%)$ & \\
\hline \multicolumn{5}{|l|}{ Sex } \\
\hline Male & $29(58 \%)$ & $11(57.1 \%)$ & $9(58.1 \%)$ & \\
\hline Female & $21(42 \%)$ & $13(42.9 \%)$ & $17(41.9 \%)$ & \\
\hline Age/median; range & $56(33-68)$ & $53(36-68)$ & $56(33-68)$ & 0.654 \\
\hline BM-infiltration (\%) median; range & $60(5-90)$ & $30(5-85)$ & $65(8-90)$ & $<0.01$ \\
\hline \multicolumn{5}{|l|}{ Ig isotype } \\
\hline IgG & $23(46.0 \%)$ & $10(41.7 \%)$ & $13(50.0 \%)$ & \\
\hline $\operatorname{Ig} \mathrm{A}$ & $12(24.0 \%)$ & $6(25.0 \%)$ & $6(23.1 \%)$ & \\
\hline $\operatorname{IgD}$ & $2(4.0 \%)$ & $2(8.3 \%)$ & $0(0.0 \%)$ & \\
\hline Light chain & $10(20.0 \%)$ & $4(16.7 \%)$ & $6(23.1 \%)$ & \\
\hline Non-secretory & $3(6.0 \%)$ & $2(8.3 \%)$ & $1(3.8 \%)$ & \\
\hline \multicolumn{4}{|l|}{ RISS stage } & 0.028 \\
\hline I & $5(10.0 \%)$ & $4(25.0 \%)$ & $1(4.3 \%)$ & \\
\hline II & $10(20.0 \%)$ & $5(31.3 \%)$ & $5(21.8 \%)$ & \\
\hline III & $24(48.0 \%)$ & $7(43.7 \%)$ & $17(73.9 \%)$ & \\
\hline \multicolumn{4}{|l|}{ DS stage } & 0.235 \\
\hline I & $1(2.0 \%)$ & $1(6.3 \%)$ & $0(0.0 \%)$ & \\
\hline II & $0(0.0 \%)$ & $0(0.0 \%)$ & $0(0.0 \%)$ & \\
\hline III & $38(76.0 \%)$ & $15(93.7 \%)$ & $23(100.0 \%)$ & \\
\hline \multicolumn{4}{|l|}{ MBD } & $<0.0001$ \\
\hline $0-2$ & $14(28.0 \%)$ & $13(54.2 \%)$ & $1(3.8 \%)$ & \\
\hline $3-4$ & $36(72.0 \%)$ & $11(45.8 \%)$ & $25(96.2 \%)$ & \\
\hline Hemoglobin $(\mathrm{g} / \mathrm{L})$ median; range & $88(44-161)$ & $109(91-161)$ & $69(44-89)$ & \\
\hline
\end{tabular}

Table 2. Baseline characteristics of 50 NDMM patients. RISS Revised international staging system, DS Durie Salmon, $M B D$ myeloma bone disease.

Cell, reagents, and antibodies. The mouse myeloma cell line 5TGM1 was kindly provided by Dr. Fenghuang Zhan (University of Arkansas for Medical Sciences, Little Rock, USA) ${ }^{14}$. and was cultured in RPMI 1640 (Invitrogen) supplemented with 10\% heat-inactivated FBS (Invitrogen), penicillin (100 IU/mL), and streptomycin $(100 \mu \mathrm{g} / \mathrm{mL})$ in a humidified incubator at $37^{\circ} \mathrm{C}$ and $5 \% \mathrm{CO}_{2} / 95 \%$ air. CD34 ${ }^{+} \mathrm{HSPCs}$ were isolated from human BM biopsies with CD34 microbeads (Miltenyi Biotec, Auburn, CA) and cultured in StemSpan SFEM containing Erythroid Expansion Supplement (StemCell Technologies). The following antibodies were purchased from BD Biosciences: CD34-APC (563), CD38-PE-Cy7 (HIT2), CD45RA-FITC (L48) and CD138-PE (MI15). The antibodies CCR1-PE (5F10B29), CCR4-PE (D8SEE), CCR5-PE (NP-6G4) and CD123-PE (6H6) were purchased from eBioscience. Antibodies against p38, phospho-p38, GATA-1, GAPDH, and HRP-conjugated secondary antibodies were purchased from Cell Signaling Technology (CST, USA). CCL3 was purchased from Sigma-Aldrich (St. Louis, MO, USA). The antagonists BX471, AZD2098 and Maraviroc were purchased from MCE (USA).

Mouse model of MM and in vivo experiments. The spontaneous syngeneic immunocompetent mouse model of MM, C57BL/KaLwRij mice ${ }^{47}$, was purchased from Harlan Laboratories Inc. (Harlan Mice, Netherlands), and the mice were housed under conditions approved by the Ethical Committee for Animal Experiments of Institute of Hematology \& Blood Diseases Hospital. Injection of the mouse MM cell line 5TGM1 can accelerate MM development. A total of $1 \times 10^{6} 5$ TGM1 cells suspended in $100 \mu \mathrm{L}$ of PBS (MM-5TGM1) or the same volume of PBS alone (Con) was injected via tail vein into C57BL/KaLwRij mice. The IgG2b level in the serum and the percentage of 5TGM1 myeloma cells in bone marrow were measured to monitor the tumour burden. Six weeks after tumour cell injection, the mice were euthanized humanely and processed to evaluate erythropoiesis in vivo. Bone marrow was collected and detected by flow cytometry as previously reported ${ }^{48}$.

Colony formation assays. Bone marrow samples were collected in heparinized tubes and processed with a Ficoll gradient. CD $34^{+}$cells were isolated using a positive magnetic bead selection protocol (Miltenyi, USA). Purified CD34 ${ }^{+}$cells were seeded in MethoCult complete medium (H4435, StemCell Technologies) following the manufacturer's instructions. For multilineage colony formation, the cells were plated in 24 -well plates in $1 \mathrm{ml}$ of $\mathrm{H} 4435$ medium at a density of $1 \times 10^{3}$ cells per well. BM plasma from MM patients was added to MethoCult complete medium at a final concentration of $100 \mu \mathrm{l} / \mathrm{ml}$. For the antagonist treatment assay, CD34 ${ }^{+}$cells were cultured in medium containing CCL3 $(50 \mathrm{ng} / \mathrm{ml})$ and the CCR1 antagonist BX471 $(1 \mu \mathrm{M})$, the CCR4 antagonist AZD2098 (100 nM), or the CCR5 antagonist maraviroc (15 nM). After 2 weeks of culture, the numbers of colonies (BFU-E, CFU-GM, CFU-GEMM) were counted. BFU-E (burst-forming unit-erythroid) colonies were identified as containing more than 200 erythroblasts in single or multiple clusters and appeared red or brown as 
the cells contain haemoglobin. CFU-GM (colony-forming unit-granulocyte, macrophage) colonies were identified as containing more than twenty granulocytes and/or macrophages without colour. CFU-GEMM (colonyforming unit-granulocyte, erythroid, macrophage, megakaryocyte) colonies were identified as containing both erythroid cells and twenty or more non-erythroid cells.

Flow cytometry analysis. Six weeks after tumour cell injection, mice were euthanized humanely. The femurs, tibias, and iliac crest were harvested immediately and placed in PBS. Bone marrow cells were obtained by rinsing femurs, tibias, and iliac crest with PBS. After collection, $1 \times 10^{6}-5 \times 10^{6}$ cells were suspended in $100 \mu \mathrm{l}$ of FACS buffer and stained with the appropriate antibodies at $4{ }^{\circ} \mathrm{C}$. According to the manufacturer's protocol, $10 \mu \mathrm{l}$ of each antibody was sequentially added to the cell suspension on ice. The workflow is shown in Supplementary Figure 1. Fresh BM cells were stained with the following antibodies: lineage antibodies (B220 (RA36B2), CD3 (17A2), Gr-1 (RB6-8C5), CD11b (M1/70), CD8a (53-6.7), CD4 (GK1.5) and Ter-119 (TER-119)), Sca-1-Pe-Cy7 (D7, BD Biosciences), c-Kit-APC (2B8), CD150-PE (TC15-12F12.2), CD48-PerCP (HM48-1, eBioscience), CD41-FITC (MWReg30), CD34-FITC (RAM34, eBioscience), CD16/32-BV421 (93), CD71-FITC (C2, BD Biosciences), CD135-PE (A2F10) and CD138-PE (281-2).

Cell cycle and apoptosis analysis. For the cell cycle analysis, cells were fixed and permeabilized using a Cytofix/Cytoperm kit (BD Biosciences), stained with anti-Ki-67 antibody, and further incubated with Hoechst $33342(20 \mu \mathrm{g} / \mathrm{ml})$. For apoptosis analysis, BM cells were stained for HSCs and then incubated with Annexin V and 7-Amino-Actinomycin D (BD Biosciences). Data were collected on a BD Canto II Flow Cytometer and analysed with FlowJo software (7.6.1).

RNA extraction and quantitative real-time PCR. Total RNA was isolated using TRIzol reagent (Invitrogen, USA). Aliquots of $1 \mu \mathrm{g}$ of total RNA were reverse transcribed using an M-MLV reverse transcriptase cDNA synthesis kit (Invitrogen, USA) as described previously ${ }^{49}$. Briefly, the cDNA and appropriate primers were mixed with SYBR Premix Ex Taq $(2 \times)$ (Takara, China), and qRT-PCR was performed using Quant Studio 5 (Thermo) in a two-step real-time PCR $\left(95^{\circ} \mathrm{C}\right.$ for $30 \mathrm{~s}$ followed by 40 cycles of $95^{\circ} \mathrm{C}$ for $5 \mathrm{~s}$ and $60^{\circ} \mathrm{C}$ for $30 \mathrm{~s}$ ). All mRNA levels of target genes were normalized to GAPDH levels and were calculated using the $2^{-\Delta \Delta C T}$ method. PCR primer sequences were as follows: GATA-1-F: 5'-cctcatccggcccaagaag-3'; GATA-1-R: 5'-atggtcagtggccggttc-3' KLF-1-F: 5'-tccatcagcacactgaccgc-3'; KLF-1-R: 5'-cggagcgccaccacttga-3'; CCL3-F: 5'-gctgactactttgagacgagc-3'; CCL3-R: 5'-ccagtccatagaagaggtagc-3'; GAPDH-F: 5'-gaaggtgaaggtcggagtc-3'; and GAPDH-R: 5'-gaagatggtgatgggatttc- $3^{\prime}$.

Western blotting. Western blotting was performed as previously described ${ }^{50}$. Briefly, protein samples were separated using SDS-PAGE and transferred onto PVDF membranes. The membranes were blocked with 5\% non-fat dry milk in TRIS-buffered saline (TBS) containing $0.05 \%$ Tween-20 (TBST) prior to incubation overnight at $4{ }^{\circ} \mathrm{C}$ with primary antibody against phosphorylated p38, total p38 or GATA1 (1:1000 dilution) overnight at $4{ }^{\circ} \mathrm{C}$. The appropriate HRP-conjugated secondary antibodies were added, and protein signals were developed with the use of enhanced chemiluminescence (ECL) reagents (Thermo Scientific). The developed images were obtained and analysed using ChemiDoc (Bio-Rad, USA).

Immunofluorescence analysis. Immunofluorescence was performed as previously described ${ }^{48}$. HSPCs were spun down on glass slides and then fixed in $-20^{\circ} \mathrm{C}$ acetone/methanol ( $1: 1$ volume) for $10 \mathrm{~min}$. Cells were blocked by immersion in $5 \%$ goat serum for $60 \mathrm{~min}$. Cells were then incubated with primary antibody against phosphorylated p38 or GATA1 (1:100 dilution in PBS containing 4\% bovine serum albumin, CST) overnight at $4{ }^{\circ} \mathrm{C}$ and incubated with the secondary antibodies Alexa Fluor 488-conjugated goat anti-mouse immunoglobulin G (IgG; H + L; 1:200, Invitrogen) or Alexa Fluor 568-conjugated goat anti-rabbit IgG at room temperature for $30 \mathrm{~min}$ in the dark. Nuclei were stained with DAPI. The slides were sealed with nail polish. Microscopic images were captured using an Ultra VIEW Vox confocal microscope (Perkin Elmer, USA).

ELISA assays. Six weeks after tumour cell or PBS injection, BM plasma and serum from peripheral blood (PB) were collected from each mouse. ELISA for CCL3 (R\&D Systems, Minneapolis, MN, USA) was performed on the serum samples and BM supernatants. A mouse IgG2b ELISA Quantitation Set (Bethyl Laboratories) and ELISA Starter Accessory Kit (Bethyl Laboratories) were used to determine Ig secretion according to the manufacturer's instructions. The results were obtained by measuring the absorbance $(450 \mathrm{~nm})$ with a microtiter plate reader, and the Ig light chain content was determined using Curve Expert (1.4) software.

RNA-seq and GSEA analysis. CD34 ${ }^{+}$cells from the bone marrow of myeloma patients and healthy donors were collected using CD34 microbeads. RNA was extracted using the Qiagen RNeasy Kit. Library construction and RNA sequencing were performed on a BGISEQ-1000 system by the Beijing Genomic Institution (www.genomics.org.cn, BGI, Shenzhen, China). Gene set enrichment analysis (GSEA) was performed with GSEA_4.0.3 (gene set enrichment analysis, Broad Institute).

Statistical analyses. Statistical analyses were performed using IBM SPSS 19.0 software and GraphPad Prism 6.0. Unpaired Student's $t$-test was used to generate $p$ values for most of the data sets. Values are represented as the mean \pm standard error of the mean (SEM) values from at least three separate experiments; ${ }^{*} p<0.05$, ${ }^{* *} p<0.01,{ }^{* *} p<0.001$, and ${ }^{* * * *} p<0.0001$. Survival analysis was performed by Kaplan-Meier analysis. 


\section{Data availability}

The data sets used and/or analysed in the current study are available from the corresponding author upon reasonable request.

Received: 12 August 2020; Accepted: 9 November 2020

Published online: 25 November 2020

\section{References}

1. Kumar, S. K. et al. Multiple myeloma. Nat. Rev. Dis. Primers 3, 17046 (2017)

2. Kawano, Y., Roccaro, A. M., Ghobrial, I. M. \& Azzi, J. Multiple myeloma and the immune microenvironment. Curr. Cancer Drug Targets 17(9), 806-818 (2017).

3. Konig, C. et al. Prevalence of iron overload vs iron deficiency in multiple myeloma: resembling or different from MDS-and stem cell transplant (SCT)-patients?. Clin. Lymphoma Myeloma Leuk. 13(6), 671-680 (2013).

4. Nemeth, E. et al. IL-6 mediates hypoferremia of inflammation by inducing the synthesis of the iron regulatory hormone hepcidin. J. Clin. Investig. 113(9), 1271-1276 (2004).

5. Goodnough, L. T., Nemeth, E. \& Ganz, T. Detection, evaluation, and management of iron-restricted erythropoiesis. Blood 116(23), 4754-4761 (2010).

6. Ludwig, H., Pohl, G. \& Osterborg, A. Anemia in multiple myeloma. Clin. Adv. Hematol. Oncol. 2(4), 233-241 (2004).

7. Morrison, S. J. \& Scadden, D. T. The bone marrow niche for haematopoietic stem cells. Nature 505(7483), 327-334 (2014).

8. Calvi, L. M. \& Link, D. C. The hematopoietic stem cell niche in homeostasis and disease. Blood 126(22), 2443-2451 (2015).

9. Kawano, Y. et al. Targeting the bone marrow microenvironment in multiple myeloma. Immunol. Rev. 263(1), 160-172 (2015).

10. Hao, M. et al. Bone marrow stromal cells protect myeloma cells from bortezomib induced apoptosis by suppressing microRNA-15a expression. Leukemia Lymphoma 52(9), 1787-1794 (2011).

11. Hao, M. et al. Suppressing miRNA-15a/-16 expression by interleukin-6 enhances drug-resistance in myeloma cells. J. Hematol. Oncol. 4, 37 (2011).

12. Sanchez-Aguilera, A. \& Mendez-Ferrer, S. The hematopoietic stem-cell niche in health and leukemia. Cell. Mol. Life Sci. CMLS 74(4), 579-590 (2017)

13. Palis, J. Primitive and definitive erythropoiesis in mammals. Front. Physiol. 5, 3 (2014).

14. Yang, Y. et al. RARalpha2 expression confers myeloma stem cell features. Blood 122(8), 1437-1447 (2013).

15. Han, J. H. et al. Macrophage inflammatory protein-1alpha is an osteoclastogenic factor in myeloma that is independent of receptor activator of nuclear factor kappaB ligand. Blood 97(11), 3349-3353 (2001).

16. Vallet, S. et al. A novel role for CCL3 (MIP-1alpha) in myeloma-induced bone disease via osteocalcin downregulation and inhibition of osteoblast function. Leukemia 25(7), 1174-1181 (2011).

17. Terpos, E., Ntanasis-Stathopoulos, I., Gavriatopoulou, M. \& Dimopoulos, M. A. Pathogenesis of bone disease in multiple myeloma: from bench to bedside. Blood Cancer J. 8(1), 7 (2018).

18. Siegel, R. L., Miller, K. D. \& Jemal, A. Cancer statistics. CA Cancer J. Clin. 70(1), 7-30 (2020).

19. Rajkumar, S. V. Multiple myeloma: every year a new standard?. Hematol. Oncol. 37(Suppl 1), 62-65 (2019).

20. Peyssonnaux, C. et al. Regulation of iron homeostasis by the hypoxia-inducible transcription factors (HIFs). J. Clin. Investig. 117(7), 1926-1932 (2007).

21. Cullis, J. Anaemia of chronic disease. Clin. Med. 13(2), 193-196 (2013).

22. Zon, L. I. Intrinsic and extrinsic control of haematopoietic stem-cell self-renewal. Nature 453(7193), 306-313 (2008).

23. Cheng, H. et al. Leukemic marrow infiltration reveals a novel role for Egr3 as a potent inhibitor of normal hematopoietic stem cell proliferation. Blood 126(11), 1302-1313 (2015).

24. Bruns, I. et al. Multiple myeloma-related deregulation of bone marrow-derived CD34(+) hematopoietic stem and progenitor cells. Blood 120(13), 2620-2630 (2012).

25. Bordini, J. et al. Erythroblast apoptosis and microenvironmental iron restriction trigger anemia in the VK ${ }^{\star} \mathrm{MYC}$ model of multiple myeloma. Haematologica 100(6), 834-841 (2015).

26. Tumburu, L. \& Thein, S. L. Genetic control of erythropoiesis. Curr. Opin. Hematol. 24(3), 173-182 (2017).

27. Ludwig, L. S. et al. Transcriptional states and chromatin accessibility underlying human erythropoiesis. Cell Rep. 27(11), 3228-3240 (2019)

28. Love, P. E., Warzecha, C. \& Li, L. Ldb1 complexes: the new master regulators of erythroid gene transcription. Trends Genet. TIG 30(1), 1-9 (2014).

29. Pinho, S. \& Frenette, P. S. Haematopoietic stem cell activity and interactions with the niche. Nat. Rev. Mol. Cell Biol. 20(5), 303-320 (2019).

30. Fields, S. Z. et al. Activin receptor antagonists for cancer-related anemia and bone disease. Exp. Opin. Investig. Drugs 22(1), 87-101 (2013).

31. Deshet-Unger, N. et al. Erythropoietin treatment in murine multiple myeloma: immune gain and bone loss. Sci. Rep. 6, 30998 (2016).

32. Hiram-Bab, S. et al. Erythropoietin directly stimulates osteoclast precursors and induces bone loss. FASEB J. 29(5), 1890-1900 (2015)

33. Palma, B. D. et al. Osteolytic lesions, cytogenetic features and bone marrow levels of cytokines and chemokines in multiple myeloma patients: role of chemokine (C-C motif) ligand 20. Leukemia 30(2), 409-416 (2016).

34. Chen, L., Wu, J., Pier, E., Zhao, Y. \& Shen, Z. mTORC2-PKBalpha/Akt1 serine 473 phosphorylation axis is essential for regulation of FOXP3 Stability by chemokine CCL3 in psoriasis. J. Invest. Dermatol. 133(2), 418-428 (2013).

35. Lentzsch, S. et al. Macrophage inflammatory protein 1-alpha (MIP-1 alpha ) triggers migration and signaling cascades mediating survival and proliferation in multiple myeloma (MM) cells. Blood 101(9), 3568-3573 (2003).

36. Wang, Y. et al. Leukemia cell infiltration causes defective erythropoiesis partially through MIP-1alpha/CCL3. Leukemia 30(9), 1897-1908 (2016)

37. Ghobrial, I. M., Detappe, A., Anderson, K. C. \& Steensma, D. P. The bone-marrow niche in MDS and MGUS: implications for AML and MM. Nat. Rev. Clin. Oncol. 15(4), 219-233 (2018).

38. McGuckin, C. P., Forraz, N. \& Liu, W. M. Diaminofluorene stain detects erythroid differentiation in immature haemopoietic cells treated with EPO, IL-3, SCF, TGFbeta1, MIP-1alpha and IFNgamma. Eur. J. Haematol. 70(2), 106-114 (2003).

39. Menu, E. et al. Role of CCR1 and CCR5 in homing and growth of multiple myeloma and in the development of osteolytic lesions: a study in the 5TMM model. Clin. Exp. Metas. 23(5-6), 291-300 (2006).

40. Dairaghi, D. J. et al. CCR1 blockade reduces tumor burden and osteolysis in vivo in a mouse model of myeloma bone disease. Blood 120(7), 1449-1457 (2012).

41. Higuchi, C. et al. Continuous inhibition of MAPK signaling promotes the early osteoblastic differentiation and mineralization of the extracellular matrix. J. Bone Miner. Res. Off. J. Am. Soc. Bone Miner. Res. 17(10), 1785-1794 (2002). 
42. Hernandez-Hernandez, A. et al. Acetylation and MAPK phosphorylation cooperate to regulate the degradation of active GATA-1. EMBO J. 25(14), 3264-3274 (2006).

43. Castellino, F. et al. Chemokines enhance immunity by guiding naive $\mathrm{CD} 8+\mathrm{T}$ cells to sites of $\mathrm{CD} 4+\mathrm{T}$ cell-dendritic cell interaction. Nature 440(7086), 890-895 (2006).

44. Cao, Q. et al. Therapeutic effect of MIP-1alpha-recruited dendritic cells on preestablished solid and metastatic tumors. Cancer Lett. 295(1), 17-26 (2010).

45. Palumbo, A. et al. Revised international staging system for multiple myeloma: a report from international myeloma working group. J. Clin. Oncol. 33(26), 2863-2869 (2015).

46. WHO: Haemoglobin Concentrations for the Diagnosis of Anaemia and Assessment of Severity (Geneva, World Health Organization 2011) (WHO/NMH/NHD/MNM/11.1)

47. Maes, K. et al. The genetic landscape of 5T models for multiple myeloma. Sci. Rep. 8(1), 15030 (2018).

48. Zang, M. et al. Cdc37 suppression induces plasma cell immaturation and bortezomib resistance in multiple myeloma via Xbp1s. Oncogenesis 9(3), 31 (2020).

49. Yu, T. et al. Polycomb-like protein 3 induces proliferation and drug resistance in multiple myeloma and is regulated by miRNA-15a. Mol. Cancer Res. 18, 1063-1073 (2020).

50. Li, Z. et al Therapeutic effects of oligo-single-stranded DNA mimicking of hsa-miR-15a-5p on multiple myeloma. Cancer Gene Ther. https://doi.org/10.1038/s41417-020-0161-3 (2020).

\title{
Acknowledgements
}

We thank Dr Fenghuang Zhan (University of Arkansas for Medical Sciences, Little Rock, USA) for kindly providing the 5TGM1 mouse myeloma cell line. We also thank Dr. Yi He of Transplantation Department and the members of the core facility of the Institute for their technical support.

\section{Author contributions}

L.L. and Z.Y. performed the research and analysed the data. X.M. and S.D. arranged the data and performed the statistical analysis. L.Q., M.H., T.C. and H.C. designed the research. L.L. and M.H. wrote the paper. W.S., X.W., C.D., J.X., W.H., S.X., G.A. and W.Z. provided clinical knowledge of M.M. J.L. and X.M. analysed the data. All the authors read and approved the final version of the manuscript.

\section{Funding}

This work was supported by the Chinese Academy of Medical Sciences (CAMS) Innovation Fund for Medical Sciences CAMS-2017-I2M-1-005, CAMS 2017-I2M-1-015 and CAMS-2016-I2M-3-031 (to M.H. and L.Q.); the Natural Science Foundation of China (81570181 \& 81400174 to M.H., 81920108006 \& 81630007 to L.Q., 81421002 $\& 81730006$ to T.C.); the Tianjin Science and Technology Supporting Program (17JCYBJC27900 to M.H.); the Non-profit Central Research Institute Fund of Chinese Academy of Medical Sciences (2018RC320012, to M.H.); CAMS Innovation Fund for Medical Sciences (CIFMS, 2016-I2M-3-023) and the Fundamental Research Funds for the Central Universities (3332020055 to L.L.).

\section{Competing interests}

The authors declare no competing interests.

\section{Additional information}

Supplementary information is available for this paper at https://doi.org/10.1038/s41598-020-77450-y.

Correspondence and requests for materials should be addressed to X.M. or M.H.

Reprints and permissions information is available at www.nature.com/reprints.

Publisher's note Springer Nature remains neutral with regard to jurisdictional claims in published maps and institutional affiliations.

\begin{abstract}
Open Access This article is licensed under a Creative Commons Attribution 4.0 International License, which permits use, sharing, adaptation, distribution and reproduction in any medium or format, as long as you give appropriate credit to the original author(s) and the source, provide a link to the Creative Commons licence, and indicate if changes were made. The images or other third party material in this article are included in the article's Creative Commons licence, unless indicated otherwise in a credit line to the material. If material is not included in the article's Creative Commons licence and your intended use is not permitted by statutory regulation or exceeds the permitted use, you will need to obtain permission directly from the copyright holder. To view a copy of this licence, visit http://creativecommons.org/licenses/by/4.0/.
\end{abstract}

(C) The Author(s) 2020 